\title{
A PROOF OF PYBER'S BASE SIZE CONJECTURE
}

\author{
HÜLYA DUYAN, ZOLTÁN HALASI, AND ATTILA MARÓTI
}

\begin{abstract}
Building on earlier papers of several authors, we establish that there exists a universal constant $c>0$ such that the minimal base size $b(G)$ of a primitive permutation group $G$ of degree $n$ satisfies $\log |G| / \log n \leq b(G)<45(\log |G| / \log n)+c$. This finishes the proof of Pyber's base size conjecture. An ingredient of the proof is that for the distinguishing number $d(G)$ (in the sense of Albertson and Collins) of a transitive permutation group $G$ of degree $n>1$ we have the estimates $\sqrt[n]{|G|}<d(G) \leq 48 \sqrt[n]{|G|}$.
\end{abstract}

\section{INTRODUCTION}

Let $G$ be a permutation group acting on a finite set $\Omega$ of size $n$. A subset $\Sigma$ of $\Omega$ is called a base for $G$ if the intersection of the stabilizers in $G$ of the elements of $\Sigma$ is trivial. Bases played a key role in the development of permutation group theoretic algorithms. For an account of such algorithms see the book of Seress [40].

It is fundamentally important to find a base of small size. For one reason this is because that leads to a reduction in the space to store elements of the permutation group. The minimal size of a base for $G$ on $\Omega$ is denoted by $b(G)$. Blaha [8] showed that the problem of finding $b(G)$ for a permutation group $G$ is NP-hard. One may approximate $b(G)$ by a greedy heuristic; always choose a point from $\Omega$ whose orbit is of largest possible size under the action of the intersection of the stabilizers in $G$ of the previous points chosen. Blaha [8] proved that the size of such a base is $O(b(G) \log \log n)$ and that this bound is sharp. (Here and throughout the paper the base of the logarithms is 2 unless otherwise stated.) On the other hand, Pyber [35] showed that there exists a universal constant $c>0$ such that almost all (a proportion tending to 1 as $n \rightarrow \infty$ ) subgroups $G$ of $\operatorname{Sym}(n)$ satisfy $b(G)>c n$.

The minimal base size of a primitive permutation group has much been investigated. Already in the nineteenth century Bochert [9] showed that $b(G) \leq n / 2$ for a primitive permutation group $G$ of degree $n$ not containing $\operatorname{Alt}(n)$. This bound was substantially

Date: September 5, 2017.

2010 Mathematics Subject Classification. 20B15, 20C99, 20B40.

Key words and phrases. minimal base size, distinguishing number, permutation group, linear group.

The second author was supported from the ERC Limits of discrete structures Grant No. 617747. The third author has received funding from the European Research Council (ERC) under the European Union's Horizon 2020 research and innovation program (grant agreement No. 648017) and was supported by the János Bolyai Research Scholarship of the Hungarian Academy of Sciences. The second and third authors were also supported by a Humboldt Return Fellowship and by National Research, Development and Innovation Office (NKFIH) Grant No. K115799. 
improved by Babai to $b(G)<4 \sqrt{n} \log n$, for uniprimitive groups $G$, in [2], and to the estimate $b(G)<2^{c \sqrt{\log n}}$ for a universal constant $c>0$, for doubly transitive groups $G$ not containing $\operatorname{Alt}(n)$, in [3]. The latter bound was improved by Pyber [34] to $b(G)<c(\log n)^{2}$ where $c$ is a universal constant. These estimates are elementary in the sense that their proofs do not require the Classification of Finite Simple Groups (CFSG). Using CFSG Liebeck [28] classified all primitive permutation groups $G$ of degree $n$ with $b(G) \geq 9 \log n$.

One of the ingredients of Liebeck's proof was a result stating that an almost simple primitive permutation group of degree $n$ in its, later called, "non-standard" action has order at most $n^{9}$. This bound was later improved by Liebeck and his result showed that the Mathieu group $M_{24}$ in its action on 24 points is the worst case. This lead Cameron and Kantor to conjecture that an almost simple primitive permutation group in its nonstandard action has bounded minimal base size, perhaps 7 with equality holding for $M_{24}$. The first part of this conjecture has been established by Liebeck and Shalev in [29] and the second half was completed in a series of papers by Cameron, Kantor [15], Liebeck, Shalev [31], [32], James [25], [26], Burness [10], Burness, Liebeck, Shalev [12], Burness, O'Brien, Wilson [13], and Burness, Guralnick, Saxl [11].

Let $d$ be a fixed positive integer. Let $\Gamma_{d}$ be the class of finite groups $G$ such that $G$ does not have a composition factor isomorphic to an alternating group of degree greater than $d$ and no classical composition factor of rank greater than $d$. Babai, Cameron, Pálfy [4] showed that if $G \in \Gamma_{d}$ is a primitive permutation group of degree $n$, then $|G|<n^{f(d)}$ for some function $f(d)$ of $d$. Babai conjectured that there is a function $g(d)$ such that $b(G)<g(d)$ whenever $G$ is a primitive permutation group in $\Gamma_{d}$. Seress [38] showed this for $G$ a solvable primitive group. Babai's conjecture was proved by Gluck, Seress, Shalev [20]. Later Liebeck, Shalev [29] showed that in Babai's conjecture the function $g(d)$ can be taken to be linear in $d$.

Since any element of a permutation group $G$ is determined by its action on a base, we clearly have $|G| \leq n^{b(G)}$ where $n=|\Omega|$ is the degree of $G$. From this we get the estimate $\log |G| / \log n \leq b(G)$. An important question of Pyber [35, Page 207] from 1993 states that for primitive permutation groups $G$ this latter bound is essentially sharp. Specifically, he asked whether there exists a universal constant $c>0$ such that

$$
b(G)<c \frac{\log |G|}{\log n} .
$$

Pyber's conjecture is an essential generalization of the known upper bounds for $b(G)$, the weaker form of the Cameron-Kantor conjecture, and Babai's conjecture.

By the Aschbacher-O'Nan-Scott theorem, primitive permutation groups fall in several types: almost simple type, diagonal type, product type, twisted wreath product type, and affine type. Pyber's conjecture has been verified for all non-affine primitive permutation groups. Work on almost simple groups has been carried out in papers towards Cameron's conjecture (see above) and by Benbenishty in [7]. Primitive permutation groups of diagonal type were treated by Gluck, Seress, Shalev [20, Remark 4.3] and Fawcett [17]. For primitive groups of product type and of twisted wreath product type the conjecture was dealt by 
Burness and Seress [14]. From these results one can deduce the general bound $b(G)<$ $45(\log |G| / \log n)$ for a non-affine primitive permutation group $G$ of degree $n$. See Section 4 for this discussion.

The most general result on affine primitive permutation groups is due to Liebeck and Shalev [30], [33] who established Pyber's conjecture in the case when a point stabilizer is a primitive linear group (see Theorem 3.1). In this paper we use a characterization of primitive linear groups of unbounded base size given by Liebeck and Shalev [30], [33] (see Theorem 3.16). There is a similar characterization of primitive linear groups of large orders due to Jaikin-Zapirain and Pyber [24, Proposition 5.7].

In case a point stabilizer of an affine primitive permutation group has order coprime to the size of its normal complement Pyber's conjecture was first established by Gluck and Magaard in [19]. Solvable or more generally, $p$-solvable affine primitive permutation groups also satisfy Pyber's conjecture (where $p$ is the prime divisor of the degree). These statements were proved by Seress [38] and Halasi and Maróti [23]. The case where the point stabilizer of an affine primitive permutation group is an imprimitive linear group has also been considered by Fawcett and Praeger in [18].

In this paper we prove the last part of Pyber's conjecture, the one on affine primitive permutation groups where the point stabilizer is an imprimitive linear group. A stronger form of Pyber's conjecture is the following.

Theorem 1.1. There exists a universal constant $c>0$ such that the minimal base size $b(G)$ of a primitive permutation group $G$ of degree $n$ satisfies

$$
\frac{\log |G|}{\log n} \leq b(G)<45 \frac{\log |G|}{\log n}+c .
$$

The minimal base size of a permutation group is related to several other invariants of the group. For example, Robinson [36] showed that if $G$ is a primitive permutation group of degree $n$ and rank $r$, then $b(G) \leq(n-1) /(r-1)$. The minimal degree of a transitive permutation group is also related to the minimal base size. There are at least two concepts termed by the name "distinguishing number". Both of these are connected to the minimal base size of a group. In 1981 Babai [2] defined the distinguishing number for a coherent configuration and used it to establish the above-mentioned bound for the minimal base size. This notion was later also used in a recent paper by Sun and Wilmes [41]. In the present paper we use a different concept labelled by the same name. This different definition was introduced for graphs in 1996 by Albertson and Collins [1] and since then many authors used it under the name "distinguishing number". For more information see Sections 2.2 and 3.4 of the excellent survey article by Bailey and Cameron [5].

For a permutation group $G$ acting on a finite set $\Omega$ we denote the minimal number of colors needed to color the elements of $\Omega$ in such a way that the stabilizer in $G$ of this coloring is trivial by $d(G)$. This invariant is called the distinguishing number of the permutation group. Seress [38] proved that $d(G) \leq 5$ for a solvable permutation group $G$. By results of Seress [39] and Dolfi [16], it follows that $d(G) \leq 4$ for a primitive permutation group $G$ of degree $n$ which does not contain $\operatorname{Alt}(n)$. If $G$ is a transitive permutation group of 
degree $n>1$, then $\sqrt[n]{|G|}<d(G)$. An equivalent form (see Theorem 2.2 and the discussion preceding it) stated by Burness and Seress [14] is that there exists a universal constant $c>0$ such that $d(G) \leq|G|^{c / n}$ provided that $G$ is a transitive permutation group of degree $n$. The proof of this latter fact misses a case. In this paper we show the following stronger result which is an ingredient of the proof of Theorem 1.1.

Theorem 1.2. Let $G$ be a transitive permutation group acting on a finite set of size $n>1$. Then $\sqrt[n]{|G|}<d(G) \leq 48 \sqrt[n]{|G|}$.

\section{The Distinguishing NUmber of A tRAnsitive PERMUtATion GROUP}

Let $G$ be a group acting on a finite set $\Omega$. A base for $G$ is a subset $\Sigma$ of $\Omega$ such that the intersection of the stabilizers in $G$ of all points in $\Sigma$ is trivial. We denote the minimal size of a base for $G$ by $b(G)$ or by $b_{\Omega}(G)$ if $\Omega$ is to be specified. More generally, for any normal subgroup $N$ of $G$ we set $b_{\Omega}(G / N)=\min \left\{k \mid \exists x_{1}, \ldots, x_{k} \in \Omega, \cap_{i=1}^{k} G_{x_{i}} \leq N\right\}$. A trivial observation is that $\max \{b(N), b(G / N)\} \leq b(G) \leq b(N)+b(G / N)$.

The purpose of this section is to study yet another invariant which is closely related to the minimal base size (see (2) of Remark 2.1).

A distinguishing partition for a finite group $G$ acting (not necessarily faithfully) on a finite set $\Omega$ is a coloring of the points of $\Omega$ in such a way that every element of $G$ fixing this coloring is contained in the kernel of the action of $G$ on $\Omega$. The minimal number of parts (or colors) of a distinguishing partition is called the distinguishing number of $G$ and is denoted by $d(G)$ or by $d_{\Omega}(G)$. Similarly as for the minimal base size above, for any normal subgroup $N$ of $G$ we define $d(G / N)$ to be the minimal number of colors needed to color the points of $\Omega$ in such a way that the stabilizer in $G$ of this coloring is contained in $N$.

\section{Remark 2.1.}

(1) For any $H \leq G$ and $N \triangleleft G$, we have $\max \{d(H), d(G / N)\} \leq d(G) \leq d(N) d(G / N)$.

(2) For the action of $G$ on the power set $P(\Omega)$ of $\Omega$ we have $b_{P(\Omega)}(G)=\lceil\log (d(G))\rceil$. More in general for the action of $G$ on the set $P^{q}(\Omega)$ of all partitions of $\Omega$ into at most $q$ parts, we have $b_{P^{q}(\Omega)}(G)=\left\lceil\log _{q}(d(G))\right\rceil$.

The main result (Theorem 1.2) of the section determines, up to a constant factor, the distinguishing number of a transitive permutation group.

By applying (2) of Remark 2.1 to Theorem 1.2, we get the following (almost) equivalent form, a slightly weaker version of which appears in [14, Theorem 3.1].

Theorem 2.2. For any transitive permutation group $G$ of degree $n>1$ we have

$$
\frac{\log |G|}{n}<b_{P(n)}(G)<7+\frac{\log |G|}{n} .
$$

In the following we aim to prove Theorem 1.2. 
Let $\Omega$ be a finite set of size $n>1$ and $G \leq \operatorname{Sym}(\Omega)$ be a (not necessarily transitive) permutation group.

For the lower bound in the statement of the theorem, notice that the action of $G$ on $\Omega$ induces an action on the set of all colorings of $\Omega$ using $d(G)$ colors and this action contains a regular orbit. Thus $|G|<d(G)^{n}$.

From now on we will prove the upper bound in the statement of Theorem 1.2.

Let us first introduce some notation which we will use throughout the paper. For a finite group $H$ acting on a set $X$ and for a subset $Y$ of $X$, we denote the setwise and the pointwise stabilizer of $Y$ in $H$ by $N_{H}(Y)$ and $C_{H}(Y)$ respectively. In the latter case when $Y=\left\{y_{1}, \ldots, y_{s}\right\}$ has size $s \geq 1$ we write $C_{H}\left(y_{1}, \ldots, y_{s}\right)$. Furthermore, for any natural number $k$, let $[k]$ denote the set $\{1,2, \ldots, k\}$.

For a system of blocks of imprimitivity $\Gamma=\left\{\Delta_{1}, \ldots, \Delta_{k}\right\}$ with $\left|\Delta_{1}\right|=\left|\Delta_{2}\right|=\ldots=$ $\left|\Delta_{k}\right|=m$ let $H_{j}=N_{G}\left(\Delta_{j}\right)$ for each $j$ with $1 \leq j \leq k$, and $N=\cap_{j=1}^{k} H_{j}$. Then $H_{j}$ acts naturally on $\Delta_{j}$ with kernel $C_{G}\left(\Delta_{j}\right)$, so $H_{j} / C_{G}\left(\Delta_{j}\right) \leq \operatorname{Sym}\left(\Delta_{j}\right)$. Furthermore, $G$ acts on $\Gamma$ with kernel $N$, so $K:=G / N \leq \operatorname{Sym}(\Gamma)$.

Our goal is to give an upper bound for the distinguishing number $d(G)=d_{\Omega}(G)$ of $G$ in terms of the distinguishing numbers $d(K)=d_{\Gamma}(K)$ of $K$ and $d\left(H_{j}\right)=d_{\Delta_{j}}\left(H_{j}\right)$ of $H_{j}$, and the degrees $k$ and $m$.

Lemma 2.3. If $H_{j}$ acts trivially on $\Delta_{j}$ (i.e. $H_{j}=C_{G}\left(\Delta_{j}\right)$ ) for every $1 \leq j \leq k$, then $d(G) \leq\lceil\sqrt[m]{d(K)}\rceil$.

Proof. The assumption of the lemma means that each orbit of $G$ on $\Omega$ has at most one common point with the block $\Delta_{j}$ for every $j \in[k]:=\{1, \ldots, k\}$. Thus, we can define a function $f: \Omega \mapsto[m]$ such that the restriction of $f$ to $\Delta_{j}$ is bijective for every $j$ and $f$ is constant on every orbit of $G$.

We define a $c=\lceil\sqrt[m]{d(K)}\rceil$-coloring $\lambda$ of $\Omega$ in the following way. Let us choose a $d(K)$ coloring $\alpha: \Gamma \mapsto\{0,1, \ldots, d(K)-1\}$ of $\Gamma$ such that only the identity of $K$ fixes $\alpha$. For every $j \in[k]$ write $\alpha\left(\Delta_{j}\right)$ in its base $c$-expansion, so

$$
\alpha\left(\Delta_{j}\right)=a_{1}(j) c^{0}+a_{2}(j) c^{1}+\ldots+a_{s+1}(j) c^{s},
$$

where $a_{1}(j), \ldots, a_{s+1}(j) \in\{0, \ldots, c-1\}$. Note that $s \leq m-1$ by the definition of $c$. If $s<$ $m-1$, let us define $a_{s+2}(j)=\ldots=a_{m}(j)=0$. Now, for any $x \in \Delta_{j}$ let $\lambda(x)=a_{f(x)}(j) \in$ $\{0, \ldots, c-1\}$. We claim that only the identity element of $G$ preserves $\lambda$. By assumption, $N=1$, so it is enough to show that if $g \in G$ fixes $\lambda$, then $g$ also fixes $\alpha$. Let $g \in G$ fixing $\lambda$ and $g\left(\Delta_{j}\right)=\Delta_{j^{\prime}}$ for some $j, j^{\prime} \in[k]$. Then we have $a_{f(x)}(j)=\lambda(x)=\lambda(g(x))=a_{f(g(x))}\left(j^{\prime}\right)$ for every $x \in \Delta_{j}$. Using the properties of $f$, this means that $a_{i}(j)=a_{i}\left(j^{\prime}\right)$ for every $i \in[m]$, i.e. $\alpha\left(\Delta_{j}\right)$ and $\alpha\left(\Delta_{j^{\prime}}\right)$ have the same base $c$-expansion.

From now on, let us assume that the action of $G$ is transitive (so $H_{j} / C_{H_{j}}\left(\Delta_{j}\right) \leq \operatorname{Sym}\left(\Delta_{j}\right)$ are permutation isomorphic for all $j \in[k])$, and $H_{1}$ acts on $\Delta_{1}$ in a primitive way. For the 
remainder of this section, we say that the action of $H_{1}$ on $\Delta_{1}$ is large if $m=\left|\Delta_{1}\right| \geq 5$ and $\operatorname{Alt}\left(\Delta_{1}\right) \leq H_{1} / C_{H_{1}}\left(\Delta_{1}\right) \leq \operatorname{Sym}\left(\Delta_{1}\right)$.

Lemma 2.4. With the above notation, if $H_{1}$ is not large, then $d(G) \leq 4 \cdot\lceil\sqrt[m]{d(K)}\rceil$.

Proof. By the results of Seress [39, Theorem 2] and Dolfi [16, Lemma 1], $d\left(H_{1}\right) \leq 4$. This means that each $\Delta_{j}$ can be colored with colors $\{0, \ldots, 3\}$ such that any element of $H_{j}$ fixing this coloring acts trivially on $\Delta_{j}$. Let $\chi: \Omega \mapsto\{0, \ldots, 3\}$ be the union of these colorings. Then Lemma 2.3 can be applied for the stabilizer of $\chi$ in $G$, so there exist a $\lceil\sqrt[m]{d(K)}\rceil$-coloring $\lambda: \Omega \mapsto\{0, \ldots,\lceil\sqrt[m]{d(K)}\rceil-1\}$ such that only the identity of $G$ fixes both colorings $\lambda$ and $\chi$. Finally, one can encode the pair $(\chi, \lambda)$ by a $4 \cdot\lceil\sqrt[m]{d(K)}\rceil$-coloring $\mu$ by choosing a suitable bijective function, e.g. let $\mu(x)=4 \cdot \lambda(x)+\chi(x)$.

It is possible to slightly modify the proof of Lemma 2.4 (still using Lemma 2.3) to allow the situation when the action of $H_{1}$ on $\Delta_{1}$ is not primitive. The modified statement is the following.

Remark 2.5. Suppose that $d\left(H_{1}\right) \leq c$ for some constant $c$ where $H_{1}$ does not necessarily act primitively on $\Delta_{1}$. Then $d(G) \leq c \cdot\lceil\sqrt[m]{d(K)}\rceil$.

Now we handle the case when the action of $H_{1}$ is large and $N \neq 1$. Then the socle of $N$ is a subdirect product of alternating groups $\operatorname{Alt}(m)$. More precisely, by [37, p. 328, Lemma], the socle of $N$ is of the form $\prod_{j} D_{j}$ where each $D_{j}$ is isomorphic to $\operatorname{Alt}(m)$ and is a diagonal subgroup of a subproduct $\prod_{\ell \in I_{j}} C_{\ell}$ where $C_{\ell} \cong \operatorname{Alt}(m)$ and the subsets $I_{j}$ form a partition of $\Gamma$ with parts of equal size. (Moreover, they form a system of blocks for $G$.) Let us denote the size of each part $I_{j}$ by $t$. In accordance with [14], we will refer to this number as the linking factor of $N$. Thus, we have $\operatorname{Alt}(m)^{k / t} \leq N \leq \operatorname{Sym}(m)^{k / t}$.

Lemma 2.6. Let us assume that $H_{1}$ is large and $N \neq 1$ with linking factor $t$. Then $d(G) \leq 3 \cdot\lceil\sqrt[t]{m}\rceil \cdot\lceil\sqrt[m]{d(K)}\rceil$

Proof. If $m=6$, then Remark 2.5 gives the result. So from now on assume that this is not the case. In what follows we will prove a slightly stronger inequality in the remaining cases, namely $d(G) \leq 2 \cdot\lceil\sqrt[t]{m}\rceil \cdot\lceil\sqrt[m]{d(K)}\rceil$.

Applying suitable bijections $\Gamma \mapsto[k]$ and $\Delta_{j} \mapsto[m]$ for every $j \in[k]$ we can identify $\Omega$ with $[m] \times[k]=\{(i, j) \mid 1 \leq i \leq m, 1 \leq j \leq k\}$ such that

$$
\begin{aligned}
& N \leq\left\{\left(\sigma_{1}, \ldots, \sigma_{k}\right) \mid \sigma_{i} \in \operatorname{Sym}([m]), \sigma_{a}=\sigma_{b} \text { if }\lceil a / t\rceil=\lceil b / t\rceil\right\}, \\
& \operatorname{soc}(N)=\left\{\left(\sigma_{1}, \ldots, \sigma_{k}\right) \mid \sigma_{i} \in \operatorname{Alt}([m]), \sigma_{a}=\sigma_{b} \text { if }\lceil a / t\rceil=\lceil b / t\rceil\right\},
\end{aligned}
$$

and the action of any $n=\left(\sigma_{1}, \ldots, \sigma_{k}\right) \in N$ on $[m] \times[k]$ is given as $n(i, j)=\left(\sigma_{j}(i), j\right)$. Under this identification, $\Delta_{j}=\{(i, j) \mid i \in[m]\}$ for every $j \in[k]$.

Let $h \in H_{j}$ for some $j=u t+v \in[k]$ where $v \in[t]$. Since $\operatorname{soc}(N) \triangleleft G$, we get that $h$ fixes the set

$$
\Omega_{u}=\Delta_{u t+1} \cup \Delta_{u t+2} \cup \ldots \cup \Delta_{u t+t}
$$


setwise. Moreover, since the restriction of $\operatorname{soc}(N)$ to $\Omega_{u}$ acts in each of $\Delta_{u t+1}, \ldots, \Delta_{u t+t}$ in the same way, and the action of $h$ on $\Omega_{u}$ must normalize this, we get that $h$ acts on $\Omega_{u}$ coordinatewise i.e. there exist $\sigma_{h} \in \operatorname{Sym}([m]), \pi_{h} \in \operatorname{Sym}([t])$ such that

$$
h(i, u t+w)=\left(\sigma_{h}(i), u t+\pi_{h}(w)\right) \text { for every } i \in[m], w \in[t] .
$$

First let us assume that $t \geq m$.

We define a 2-coloring $\chi$ of $\Omega=[m] \times[k]$ as

$$
\chi(i, j)=\left\{\begin{array}{ll}
1 & \text { if } i \leq j(\bmod t) \leq m \\
0 & \text { if } i>j(\bmod t) \text { or } j(\bmod t)>m
\end{array} .\right.
$$

That is, each $\Omega_{u}$ is colored in the same way; only the first $w$ elements of $\Delta_{u t+w}$ are colored with 1 , unless $w>m$ when no element of $\delta_{u t+w}$ is colored with 1 . (Notice that if $j$ is a multiple of $t$ then here $j(\bmod t)$ means $t($ not 0$)$.)

Now, let $h \in H_{j}$ for some $j=u t+v, v=j(\bmod t)$ preserving $\chi$. If the action of $h$ on $\Omega_{u}$ is given by $\left(\sigma_{h}, \pi_{h}\right) \in \operatorname{Sym}([m]) \times \operatorname{Sym}([t])$, then $\sigma_{h}$ must fix each set $[w], w \in[m]$, i.e. $\sigma_{h}=\mathrm{id}_{[m]}$. It follows that $h \in H_{j}$ acts trivially on $\Delta_{j}$. So, Lemma 2.3 can be applied to the stabilizer of $\chi$ in $G$ to get a $\lceil\sqrt[m]{d(K)}\rceil$-coloring $\lambda$ of $\Omega$ such that only the identity element of $G$ preserves both $\chi$ and $\lambda$. Finally, as in the last paragraph of the previous lemma, the pair $(\chi, \lambda)$ can be encoded with the $2\lceil\sqrt[m]{d(K)}\rceil$-coloring $\mu(x):=2 \cdot \lambda(x)+\chi(x)$.

Now, let $t<m$. First we define a 2-coloring $\chi$ of $\Omega=[m] \times[k]$ in a similar way as for the previous case:

$$
\chi(i, j)=\left\{\begin{array}{ll}
1 & \text { if } i \leq j(\bmod t) \\
0 & \text { if } i>j(\bmod t)
\end{array} .\right.
$$

If $h \in H_{j}$ for some $j=u t+v, v \equiv j(\bmod t)$ preserving $\chi$, then $h \in \cap_{w=1}^{t} H_{u t+w}$ must hold. Moreover, the action of $h$ on each $\Delta_{u t+w}$ must be the same.

Second, we can define a $\lceil\sqrt[t]{m}\rceil$-coloring $\beta_{u}: \Omega_{u} \mapsto\{0, \ldots,\lceil\sqrt[t]{m}-1\}$ for every $u$ such that if $h \in H_{u t+v}$ fixes both $\chi$ and $\beta_{u}$, then it acts trivially on $\Omega_{u}$. This construction is analogous to the construction of $\lambda$ given in the proof of Lemma 2.3. In fact, one can use Lemma 2.3 directly by observing that $\left\{\Lambda_{i}=\{(i, u t+w) \mid w \in[t]\}\right\}_{i}$ is a system of blocks of imprimitivity of the stabilizer $T_{j}$ of $\chi$ in $H_{j}$ and the setwise stabilizer of each $\Lambda_{i}$ in $T_{j}$ acts trivially on $\Lambda_{i}$. Let $\beta: \Omega \mapsto\left\{0, \ldots,\lceil\sqrt[t]{m}-1\}\right.$ be the union of the $\beta_{u}$. Thus, we get that Lemma 2.3 can be applied for the intersections of the stabilizers of $\chi$ and $\beta$. Thus, there is a $\lceil\sqrt[m]{d(K)}\rceil$-coloring $\lambda: \Omega \mapsto\{0, \ldots,\lceil\sqrt[m]{d(K)}\rceil-1\}$ such that only the identity element of $G$ fixes all of the colorings $\chi, \beta, \lambda$. Finally, we can encode the triple $(\chi, \beta, \lambda)$ with the $2 \cdot\lceil\sqrt[t]{m}\rceil \cdot \sqrt[m]{d(K)}\rceil$-coloring $\mu$ of $\Omega$ given as $\mu(x):=2 \cdot\lceil\sqrt[t]{m} \lambda(x)+2 \cdot \beta(x)+\chi(x)$.

A permutation group $G \leq \operatorname{Sym}(\Omega)$ is called quasi-primitive if every non-trivial normal subgroup of $G$ is transitive on $\Omega$. Clearly, every primitive permutation group is quasiprimitive.

Lemma 2.7. If $G \leq \operatorname{Sym}(\Omega)$ is a (finite) quasi-primitive permutation group, then $d(G) \leq 4$ or $\operatorname{Alt}(\Omega) \leq G \leq \operatorname{Sym}(\Omega)$. 
Proof. Let us prove the lemma by induction on $n=|\Omega|$. If $G$ is a primitive permutation group, then the claim follows by Seress [39, Theorem 2] and Dolfi [16, Lemma 1]. Suppose that $G$ is not primitive but quasi-primitive. Let $\Gamma$ be a system of blocks for $G$ with $k=|\Gamma|<n$ maximal. Let $K \cong G$ be the action of $G$ on $\Gamma$. Since a distinguishing partition of $\Gamma$ for $K$ gives rise naturally to a distinguishing partition of $\Omega$ for $G$, we have $d_{\Omega}(G) \leq d_{\Gamma}(K)$. By induction, $d(G) \leq d(K) \leq 4$ or $\operatorname{Alt}(\Gamma) \leq K \leq \operatorname{Sym}(\Gamma)$. Thus we may assume that $\operatorname{Alt}(k) \leq G \leq \operatorname{Sym}(k)$ with $k \geq 5$. Each element of $\Gamma$ is a block of size at least $k-1$. For each $i$ with $0 \leq i \leq k-1$ color $i$ letters in block $i+1$ with 1 and the rest 0 . This way we colored the elements of $\Omega$ with 2 colors in such a way that the stabilizer in $G$ of this coloring is trivial. Thus $d(G) \leq 2$.

A permutation group is defined to be innately transitive if there is a minimal normal subgroup of the group which is transitive. Such groups were introduced and studied by Bamberg and Praeger [6]. A quasi-primitive permutation group is innately transitive. The next theorem is a generalization of Lemma 2.7. It considers a class of groups which contains the class of innately transitive groups.

Theorem 2.8. Let $M \triangleleft G \leq \operatorname{Sym}(\Omega)$ be transitive permutation groups where $M$ is a direct product of isomorphic simple groups. Then $d(G) \leq 12$ or $\operatorname{Alt}(\Omega) \leq G \leq \operatorname{Sym}(\Omega)$.

Proof. We prove the claim using induction on $n=|\Omega|$. By Lemma 2.7 we may assume that $G$ is not a quasi-primitive permutation group.

As before, let $\Gamma=\left\{\Delta_{1}, \ldots, \Delta_{k}\right\}$ be a system of imprimitivity consisting of minimal blocks, each of size $m$, for the action of $G$. Let the kernel of the action of $G$ on $\Gamma$ be $N$ and set $K=G / N$, a subgroup of $\operatorname{Sym}(\Gamma)$.

We claim that we may assume that $N \neq 1$. Suppose $N=1$. By the induction hypothesis, $d(G) \leq d_{\Omega}(G) \leq d_{\Gamma}(K) \leq 12$, or $G \cong \operatorname{Alt}(\Gamma)$ or $G \cong \operatorname{Sym}(\Gamma)$ with $k \geq 13$. In the latter case $G$ is quasi-primitive, since $M=\operatorname{soc}(G)$ is transitive. The claim follows.

We claim that we may assume that the action of $H_{1}$ on $\Delta_{1}$ is large. For assume that the action of $H_{1}$ on $\Delta_{1}$ is not large. By the induction hypothesis, we know that $d(K) \leq 12$ or $K$ is an alternating or symmetric group of degree at least 13 in its natural action on $\Gamma$. In the previous case $d(G) \leq 12$ follows by use of Lemma 2.4 (for $m \geq 3$ ) and Remark 2.5 (for $m=2)$. Suppose that the latter case holds. If $m \geq k-1$, then Lemma 2.4 gives $d(G) \leq 8$. Suppose that $m<k-1$. Notice that since $M$ must act transitively on $\Gamma$, is normal in $G$ and is the direct product of isomorphic simple groups, we see that $M$ is a direct product of copies of $\operatorname{Alt}(k)$. Since $m<k-1$, the stabilizer of $\Delta_{1}$ in $M$ acts trivially on $\Delta_{1}$, and this contradicts the transitivity of $M$.

Since the action of $H_{1}$ on $\Delta_{1}$ is non-empty (that is, $N \neq 1$ ) and large, $R=\operatorname{Soc}(N)$ is isomorphic to a direct product of, say $r$ copies of $\operatorname{Alt}(m)$ where $m \geq 5$ (see [37, p. 328, Lemma]). Furthermore, since $G$ acts transitively on $\Gamma$, the normal subgroup $R$ of $G$ is in fact a minimal normal subgroup of $G$. 
We claim that $R \leq M$. Suppose otherwise. Then $R \cap M=1$ implies that $R$ is contained in the centralizer $C$ of $M$ in $\operatorname{Sym}(\Omega)$. Since $M$ is transitive, $C$ must be semiregular. However $R$ is not semiregular. Thus $R \leq M$.

In fact, $R<M$ since $M$ is transitive on $\Gamma$ and $R$ is not. Furthermore, since $R$ and so $M$ is a direct product of copies of $\operatorname{Alt}(m)$, we must have $k \geq m$. By the fact that $M$ acts transitively on $\Gamma$, it also follows that $M$ acts transitively on the set of $r$ direct factors of $R$. But every subnormal subgroup of $M$ is also normal in $M$, which forces $r=1$ and so $t=k$.

By Lemma 2.6, $d(G) \leq 3 \cdot\lceil\sqrt[k]{m}\rceil \cdot\lceil\sqrt[m]{d(K)}\rceil=6 \cdot\lceil\sqrt[m]{d(K)}\rceil$. By the induction hypothesis, $d(K) \leq 12$ (in which case $d(G) \leq 12$ by the previous inequality) or $K$ is an alternating or a symmetric group of degree $k \geq 13$. But in the latter case $m=k$ (and $d(K) \leq m$ ). Thus $\lceil\sqrt[m]{d(K)}\rceil=2$ and so $d(G) \leq 12$ by Lemma 2.6.

Proof of Theorem 1.2. First suppose that $G \leq \operatorname{Sym}(\Omega)$ is a quasi-primitive permutation group. By Lemma 2.7, we may assume that $n=|\Omega| \geq 48$ and $\operatorname{Alt}(\Omega) \leq G \leq \operatorname{Sym}(\Omega)$. In this case we have $d(G) \leq n<48 \sqrt[n]{n ! / 2}$ where the second inequality follows from the fact that $\frac{1}{2}(n / 3)^{n}<n ! / 2$. Thus we may assume that $G \leq \operatorname{Sym}(\Omega)$ is not a quasi-primitive permutation group.

Let $M$ be a minimal normal subgroup in $G$ which does not act transitively on $\Omega$. Let an orbit of $M$ on $\Omega$ be $\Sigma$, and let $\Gamma$ be the set of orbits of $M$ on $\Omega$. Let the size of $\Gamma$ be $k$ and let $H$ be the stabilizer in $G$ of $\Sigma$. As before, denote the distinguishing number of $H$ acting on $\Sigma$ by $d_{\Sigma}(H)$. Since $M \triangleleft H$, Theorem 2.8 implies that $d_{\Sigma}(H) \leq 12$ or $\operatorname{Alt}(\Sigma) \leq H / C_{H}(\Sigma) \leq \operatorname{Sym}(\Sigma)$.

Case $1 . d_{\Sigma}(H) \leq 12$.

By Remark 2.5, $d(G) \leq 12\lceil\sqrt[m]{d(K)}\rceil$ where $K$ is the action of $G$ on $\Gamma$ and $m=|\Sigma|$. Since $K$ is a transitive group on $k$ points, by induction we have $d(K) \leq 48 \sqrt[k]{|K|}$. If $m \geq 6$, then

$$
d(G) \leq 12\lceil\sqrt[m]{d(K)}\rceil \leq 12\lceil\sqrt[m]{48 \sqrt[k]{|K|} \mid} \leq 24 \sqrt[m]{48 \sqrt[k]{|K|}} \leq 48 \sqrt[n]{|K|} \leq 48 \sqrt[n]{|G|}
$$

If $m \leq 5$ then we can use the previous estimate with 12 replaced by $m$ and 24 replaced by $2 m$.

Case 2. $\operatorname{Alt}(\Sigma) \leq H / C_{H}(\Sigma) \leq \operatorname{Sym}(\Sigma)$ with $|\Sigma|=m \geq 13$.

In this case the action of $H$ on $\Sigma$ is large. Let the kernel of the action of $G$ on $\Gamma$ be $N$. Since $M \leq N$, we know that $N \neq 1$. Set $\epsilon=1$ if $t=1$ and $\epsilon=2$ if $t \neq 1$. We have the following by Lemma 2.6 (and its notation).

$$
d(G) \leq 3\lceil\sqrt[t]{m}\rceil\lceil\sqrt[m]{d(K)}\rceil \leq 6 \epsilon \sqrt[t]{m} \sqrt[m]{d(K)}=6 \epsilon \sqrt[m k]{m^{m k / t}} \sqrt[m]{d(K)}
$$


Set $c=6 \cdot 2^{1 / m t} \cdot 3^{1 / t}$. By use of the inequality $\frac{1}{2}(m / 3)^{m}<m ! / 2=|\operatorname{Alt}(m)|$, we have that $d(G)$ is at most

$$
6 \epsilon \sqrt[m k]{m^{m k / t}} \sqrt[m]{d(K)}<6 \epsilon \sqrt[m k]{\left((m ! / 2) \cdot 2 \cdot 3^{m}\right)^{k / t}} \sqrt[m]{d(K)} \leq c \cdot \epsilon \sqrt[n]{(|\operatorname{Alt}(m)|)^{k / t}} \sqrt[m]{d(K)}
$$

By the paragraph after Remark 2.5, we know that $\operatorname{Alt}(m)^{k / t} \leq N$. This gives the inequality $d(G)<c \cdot \epsilon \sqrt[n]{|N|} \sqrt[m]{d(K)}$. By the induction hypothesis, we have $d(K) \leq 48 \sqrt[k]{|K|}$. Thus

$$
d(G)<c \cdot \epsilon \sqrt[m]{48} \sqrt[n]{|N|} \sqrt[n]{|K|} \leq 6 \cdot \epsilon \cdot 2^{1 / 13 t} 3 \sqrt[1 / t]{13} \sqrt[n]{|G|}<48 \sqrt[n]{|G|}
$$

\section{The AFFine CASE}

3.1. Some reductions and notation. We begin our study of Theorem 1.1 in the case of affine primitive permutation groups.

Let $G$ be an affine primitive permutation group acting on a finite set $\Omega$. Then $G$ contains a unique minimal normal subgroup $V$ acting regularly on $\Omega$, so $|\Omega|=p^{d}$ for some prime $p$ and it can be identified with the finite vector space $V$ over $\mathbb{F}_{p}$ of dimension $d$. Furthermore, $G=V \rtimes H$ for some $H \leq G L(V)$ and $H$ acts faithfully and irreducibly on the vector space $V$. Clearly, $b(G)=b_{V}(G)=b_{V}(H)+1$.

In this section we will show that there exists a universal constant $c>0$ such that for the affine primitive permutation group $G=V \rtimes H$, we have

$$
b_{V}(H) \leq 45(\log |H| / \log |V|)+c .
$$

The following theorem shows that we may assume that $H$ acts imprimitively (and irreducibly) on $V$.

Theorem 3.1 (Liebeck, Shalev [30], [33]). There exists a universal constant $c>0$ such that if $H$ acts primitively on $V$, then $b_{V}(H) \leq \max \{18(\log |H| / \log |V|)+30, c\}$.

Thus we may assume that $V$ is an imprimitive irreducible $\mathbb{F}_{p} H$-module. Let $V=\oplus_{i=1}^{t} V_{i}$ be a decomposition of $V$ into a sum of subspaces $V_{i}$ of $V$ that is preserved by the action of $H$. For every $i$ with $1 \leq i \leq t$, let $H_{i}=N_{H}\left(V_{i}\right)$ and let $K_{i}=H_{i} / C_{H_{i}}\left(V_{i}\right) \leq G L\left(V_{i}\right)$ be the image of the restriction of $H_{i}$ to $V_{i}$. The group $H$ acts on the set $\Pi=\left\{V_{1}, \ldots, V_{t}\right\}$ in a transitive way. Let $N$ be the kernel of this action and let $P$ be the image of $H$ in $\operatorname{Sym}(\Pi)$. So $N=\cap_{i=1}^{t} H_{i}$ and $P=H / N$.

As an easy application of the results of Section 2, we first prove Theorem 1.1 in the case when each $b_{V_{i}}\left(K_{i}\right)$ is bounded (see Theorem 3.4). Note that because the action of $P$ on $\Pi$ is transitive, it is enough to assume this only for $K_{1}$. First we handle the even more special case when $K_{1}$ is trivial.

Lemma 3.2. If $K_{1}=1$, then $b_{V}(H)=\left\lceil\log _{\left|V_{1}\right|} d_{\Pi}(P)\right\rceil$. 
Proof. First note that the condition $K_{1}=1$ implies that every orbit of $H$ in $\cup_{i=1}^{t} V_{i}$ contains exactly one element from every subspace $V_{i}$, which defines a one-to-one correspondence $\alpha_{i j}: V_{i} \mapsto V_{j}$ between any pair of subspaces $V_{i}$ and $V_{j}$.

Let $b$ be a positive integer. Let $w_{s}=v_{s}^{(1)}+v_{s}^{(2)}+\ldots+v_{s}^{(t)}$ be vectors in $V$ for $1 \leq s \leq b$ decomposed with respect to the direct sum decomposition $V=\oplus_{i} V_{i}$. We define an equivalence relation on $\Pi$ by $V_{i} \sim V_{j}$ if and only if $\left(v_{1}^{(i)}, \ldots, v_{b}^{(i)}\right)$ corresponds to $\left(v_{1}^{(j)}, \ldots, v_{b}^{(j)}\right)$, i.e. $\alpha_{i j}\left(v_{s}^{(i)}\right)=v_{s}^{(j)}$ for every $1 \leq s \leq b$. Then the set $\left\{w_{1}, \ldots, w_{b}\right\}$ is a base for $H$ on $V$ if and only if $\sim$ defines a distinguishing partition for $P$ on $\Pi$. The number of different vectors of the form $\left(v_{1}^{(i)}, \ldots, v_{b}^{(i)}\right)$ with entries from $V_{i}$ (for any $i$ ) is $\left|V_{1}\right|^{b}$. It follows that $b_{V}(H)$ is the smallest integer such that $\left|V_{1}\right|^{b_{V}(H)}$ is at least $d_{\Pi}(P)$.

Remark 3.3. Note that this proof also works if $P$ is not transitive on $\Pi$ but $K_{i}=1$ for every $i$ with $1 \leq i \leq t$.

Theorem 3.4. Let us assume that $b_{V_{1}}\left(K_{1}\right) \leq b$ for some constant $b$. Then we have

$$
b_{V}(H) \leq b+1+\log 48+\frac{\log |P|}{\log |V|} .
$$

Proof. By our assumption, for each $1 \leq i \leq t$ we can choose a base $\left\{v_{1}^{(i)}, v_{2}^{(i)}, \ldots, v_{b}^{(i)}\right\} \subset V_{i}$ for $K_{i} \simeq H_{i} / C_{H_{i}}\left(V_{i}\right)$. Put $w_{s}=\sum_{i=1}^{t} v_{s}^{(i)}$ for every $1 \leq s \leq b$ and let $L=\cap_{s} C_{H}\left(w_{s}\right)$. Then $L \cap H_{i}=C_{L}\left(V_{i}\right)$ for every $i$ so we can apply Lemma 3.2 for $L$ (see also Remark 3.3). Hence $b_{V}(H) \leq b+\left\lceil\log _{\left|V_{1}\right|} d_{\Pi}(P)\right\rceil$. Since $d_{\Pi}(P) \leq 48 \sqrt[t]{|P|}$ by Theorem 1.2, we get

$$
b_{V}(H) \leq b+1+\log _{\left|V_{1}\right|}(48 \sqrt[t]{|P|}) \leq b+1+\log 48+\frac{\log |P|}{t \log \left|V_{1}\right|}=b+1+\log 48+\frac{\log |P|}{\log |V|},
$$

as claimed.

Note that Theorem 3.4 proves Theorem 1.1 in case $b+1+\log 48$ is bounded. In other words, we must now look at situations when $b_{V_{1}}\left(K_{1}\right)$ is not bounded by any fixed constant.

For the remainder of this section, it will be more convenient for us to use the language of group representations. So, instead of choosing $H$ as a fixed linear subgroup of $G L(V)$, let $H$ be a fixed abstract group and $X: H \rightarrow G L(V)$ a representation of $H$. Then we would like to give an upper bound for $b_{V}(X(H))$. (The reason for this is that in the proof, we will reduce this problem to some other representations of $H$ with simpler image structure.) Moreover, in order to use a theorem of Liebeck and Shalev [33, Theorem 1], we need also extend the base field to consider vector spaces over $\mathbb{F}_{q}$ for $q$ being a power of $p$. (Of course, the base size $b_{V}(X(H))$ is independent on whether we view $V$ as an $\mathbb{F}_{p}$-space or as an $\mathbb{F}_{q}$-space.) Occasionally, we want to view the vector space $V$ over $\mathbb{F}_{q}$ as a vector space over $\mathbb{F}_{p}$, which we will emphasize by the notation $V(p)$.

By using our previous notation, we assume that $V=\oplus_{i=1}^{t} V_{i}$ is a direct sum of $\mathbb{F}_{q}$-spaces and $X: H \rightarrow G L(V)$ is a representation such that $X(H)$ permutes the set $\Pi=\left\{V_{1}, \ldots, V_{t}\right\}$ 
in a transitive way. Thus, the representation $X$ is equivalent to the induced representation $\operatorname{Ind}_{H_{1}}^{H}\left(X_{1}\right)$, where $X_{1}: H_{1} \rightarrow G L\left(V_{1}\right)$ is a linear representation of $H_{1}$.

In Sections 3.2 and 3.3 we first consider two special cases, which we will respectively call alternating-induced and classical-induced classes. Here alternating-induced means that $K_{1}$ is isomorphic to an alternating or symmetric group, and $V_{1}$ as an $\mathbb{F}_{q} K_{1}$-module is the deleted permutation module for $K_{1}$. Similarly, classical-induced means that $K_{1}$ is a classical group (maybe over some subfield $\mathbb{F}_{q_{0}} \leq \mathbb{F}_{q}$ ) with its natural action on $V_{1}$. Then we show in Section 3.4 how the general case can be reduced to one of these modules.

In fact, in order to be able to use a reduction argument in Section 3.4, we need to work with the following natural generalization of projective representations.

Definition 3.5. Let $V$ be a finite vector space over $\mathbb{F}_{q}$ and $T \leq G L(V)$ any subgroup. We say that a map $X: H \rightarrow G L(V)$ is a $(\bmod T)$-representation of $H$ if the following two properties hold:

(1) $X(g)$ normalizes $T$ for every $g \in H$;

(2) $X(g h) T=X(g) X(h) T$ for every $g, h \in H$.

Definition 3.6. Let $T \leq G L(V)$ and $X_{1}, X_{2}: H \rightarrow G L(V)$ be two $(\bmod T)$-representations of $H$. We say that $X_{1}$ and $X_{2}$ are $(\bmod T)$-equivalent if there is an $f \in N_{G L(V)}(T)$ such that $X_{1}(g) T=f X_{2}(g) f^{-1} T$ for all $g \in G$.

For a $(\bmod T)$-representation $X: H \rightarrow G L(V)$, we define the corresponding base size of $H$ as $b_{X}(H):=b_{V}(X(H) T)$ (note that $X(H) T$ is a subgroup of $G L(V)$ ). It is easy to see that equivalent $(\bmod T)$-representations have the same base size. Note that $b_{V}(H) \leq b_{X}(H)$ in case $H \leq G L(V)$ and $X=$ id.

For $T=1$ a $(\bmod T)$-representation is the same as a linear representation.

In this paragraph let $T=Z(G L(V)) \simeq \mathbb{F}_{q}^{\times}$be the group of all scalar transformations on $V$. Then a $(\bmod T)$-representation of $H$ is the same as a projective representation of $H$. Furthermore, in this case $T$-equivalence of two $T$-representations of $H$ means exactly that they are projectively equivalent. Slightly more generally, a map $X: H \rightarrow G L(V(p))$ satisfies (1) of Definition 3.5 if and only if $H$ is mapped to $\Gamma L(V)$. In the following, we will also call the $(\bmod T)$-representation $X: H \rightarrow \Gamma L(V)$ (sometimes we allow the codomain of a $(\bmod T)$-representation to be $\Gamma L(V))$ a projective representation. Furthermore, for any projective representation $X: H \rightarrow \Gamma L(V)$, we will also denote by $X$ the associated homomorphism $H \rightarrow P \Gamma L(V)$ (which we again call a projective representation).

For the remainder, we consider the special case when $V=\oplus_{i=1}^{t} V_{i}$ is a direct sum of $\mathbb{F}_{q^{-} \text {-spaces, and }}$

$$
T_{V}=\left\{g \in G L(V) \mid g\left(V_{i}\right)=V_{i} \text { and }\left.g\right|_{V_{i}} \in Z\left(G L\left(V_{i}\right)\right) \quad \forall 1 \leq i \leq t\right\} \simeq\left(\mathbb{F}_{q}^{\times}\right)^{t} .
$$

If a direct sum decomposition of a vector space $U$ is given, then $T_{U}$ will always denote the appropriate subgroup defined by the above displayed formula. 
If $X: H \rightarrow G L(V)$ is an arbitrary map, then $X$ satisfies (1) of Definition 3.5 (with $\left.T=T_{V}\right)$ if and only if the direct sum decomposition $V=\oplus_{i=1}^{t} V_{i}$ is preserved by $X(H)$. In particular, if $X$ happens to be a linear representation of $H$ preserving the direct sum decomposition $V=\oplus_{i=1}^{t} V_{i}$, then $X$ is also a $\left(\bmod T_{V}\right)$-representation of $H$.

A further observation is that if $X: H \rightarrow G L(V(p))$ is a $\left(\bmod T_{V}\right)$-representation, then the restricted map $X_{i}: H_{i} \rightarrow G L\left(V_{i}\right)$ is a projective representation of $H_{i}$. (Here $X_{i}$ is defined so that first we take the restriction of $X$ to $H_{i}$, then we restrict the action of $X\left(H_{i}\right)$ to $V_{i}$.) Conversely, if $X_{1}: H_{1} \rightarrow \Gamma L\left(V_{1}\right)$ is any projective representation, then the induced representation $X=\operatorname{Ind}_{H_{1}}^{H}\left(X_{1}\right): H \rightarrow G L(V(p))$ will be a $\left(\bmod T_{V}\right)$-representation of $H$ transitively permuting the $V_{i}$, and it is easy to see that every $\left(\bmod T_{V}\right)$-representation of $H$ transitively permuting the $V_{i}$ can be obtained in this way. Here the induced representation $X=\operatorname{Ind}_{H_{1}}^{H}\left(X_{1}\right)$ can be defined with the help of a transversal in $H$ to $H_{1}$, so it is not uniquely defined. However, it is uniquely defined up to $\left(\bmod T_{V}\right)$-equivalence, so this will not be a problem for us.

So, for the remainder, we assume that the groups $H_{1} \leq H$ are fixed, and we consider representations of the form $X=\operatorname{Ind}_{H_{1}}^{H}\left(X_{1}\right)$, where $X_{1}: H_{1} \rightarrow \Gamma L\left(V_{1}\right)$ is a projective representation of $H_{1}$.

3.2. Alternating-induced representations. In this subsection assume that for all $i$ with $1 \leq i \leq t$, the groups $K_{i} \leq G L\left(V_{i}\right)$ are isomorphic to some alternating or symmetric group of degree $k$ at least 7 , and $K_{i}$ acts on $V_{i}$ such that $V_{i}$ as an $\mathbb{F}_{q} K_{i}$-module ( $q$ is a power of $p$ ) is isomorphic to the non-trivial irreducible component of the permutation module obtained by the natural permutation action of $K_{i}$ on a fixed basis of a vector space of dimension $k$ over $\mathbb{F}_{q}$. In this situation we say that $V \simeq \operatorname{Ind}_{H_{1}}^{H}\left(V_{1}\right)$ is an alternating-induced $\mathbb{F}_{q} H$-module, and $H \leq G L(V)$ is an alternating-induced group.

In the following proposition we describe the construction of the module $V_{i}$.

Proposition 3.7. Let $K \simeq \operatorname{Alt}(k)$ or $\operatorname{Sym}(k)$ and consider its action on an $\mathbb{F}_{q}$ vector space $U$ of dimension $k \geq 5$, defined by permuting the elements of a fixed basis $\left\{e_{1}, \ldots, e_{k}\right\}$ of $U$. Let us define the subspaces

$$
U_{0}=\left\{\sum_{i} \alpha_{i} e_{i} \mid \alpha_{i} \in \mathbb{F}_{q}, \sum_{i} \alpha_{i}=0\right\} \quad \text { and } \quad W=\left\{\alpha\left(\sum_{i} e_{i}\right) \mid \alpha \in \mathbb{F}_{q}\right\} .
$$

(1) If $p \nmid k$, then $U=U_{0} \oplus W, W$ is isomorphic to the trivial $\mathbb{F}_{q} K$-module and $U_{0}$ is the unique non-trivial irreducible component of the $\mathbb{F}_{q} K$-module $U$.

(2) If $p \mid k$, then $U \geq U_{0} \geq W$, both $U / U_{0}$ and $W$ are isomorphic to the trivial $\mathbb{F}_{q} K$-module and $U_{0} / W$ is the unique non-trivial irreducible component of the $\mathbb{F}_{q} K$ module $U$.

Proof. This can be derived from [27, Page 185].

We can apply Proposition 3.7 to each pair $K_{i}, V_{i}$ to define $\mathbb{F}_{q} K_{i}$-modules $U_{i}$ and their submodules $U_{i, 0}, W_{i} \leq U_{i}$ such that either $V_{i} \simeq U_{i, 0}($ for $p \nmid k)$ or $V_{i} \simeq U_{i, 0} / W_{i}($ for $p \mid k)$. 
Then the original action of $H$ on $V$ may be defined using the action of $H$ on $U:=\oplus_{i} U_{i}$. Moreover, if we choose a basis $\left\{e_{1}^{(i)}, \ldots, e_{k}^{(i)}\right\} \subset U_{i}$ for every $i$ as in Proposition 3.7 in a suitable way, then $\left\{e_{j}^{(i)} \mid 1 \leq i \leq t, 1 \leq j \leq k\right\}$ will be a basis of $U$ such that $H$ acts on $U$ by permuting the elements of this basis.

The next lemma says that $b_{V}(H)$ is bounded by a linear function of $b_{U}(H)$.

Lemma 3.8. With the above notation $b_{V}(H) \leq 2 b_{U}(H)+3$ for $k \geq 7$.

Proof. First, we define three vectors $w_{1}, w_{2}, w_{3} \in U_{1,0} \oplus U_{2,0} \oplus \ldots \oplus U_{t, 0}$ as linear combinations of the basis vectors $\left\{e_{j}^{(i)} \mid 1 \leq i \leq t, 1 \leq j \leq k\right\}$ as follows.

$$
w_{1}=\sum_{i=1}^{t}\left(e_{1}^{(i)}-e_{2}^{(i)}\right), w_{2}=\sum_{i=1}^{t}\left(e_{2}^{(i)}-e_{3}^{(i)}\right), w_{3}=\sum_{i=1}^{t}\left(e_{3}^{(i)}-e_{4}^{(i)}\right) .
$$

Let $L=C_{H}\left(w_{1}, w_{2}, w_{3}\right)$, so $\left\{e_{j}^{(i)} \mid 1 \leq i \leq t\right\}$ are $L$-invariant subsets for $1 \leq j \leq 4$.

Let $\left\{u_{1}, \ldots, u_{b}\right\} \subset U$ be a base for $H$ of size $b=b_{U}(H)$. Now, for any $u \in\left\{u_{1}, \ldots, u_{b}\right\}$ we define two further vectors $u^{e}, u^{f} \in U_{1,0} \oplus U_{2,0} \oplus \ldots \oplus U_{t, 0}$ as follows. Write $u=\sum_{i, j} a_{i j} e_{j}^{(i)}$ and define

$$
\begin{aligned}
& u^{e}=\sum_{i} \sum_{j>2} a_{i j} e_{j}^{(i)}+\sum_{i} \beta_{i} e_{1}^{(i)}, \text { for } \beta_{i}=-\sum_{j>2} a_{i j}, \\
& u^{f}=\sum_{i} \sum_{j \leq 2} a_{i j} e_{j}^{(i)}+\sum_{i} \gamma_{i} e_{3}^{(i)}, \text { for } \gamma_{i}=-\left(a_{i 1}+a_{i 2}\right) .
\end{aligned}
$$

The above definition of the $\beta_{i}$ and $\gamma_{i}$ ensures that the projection of $u^{e}$ and $u^{f}$ to any $U_{i}$ is really in $U_{i, 0}$. Furthermore, if $l \in L$ fixes $u^{e}$, then because of the above mentioned $L$ invariant subsets of basis vectors we get that $l$ must fix both $\sum_{i} \beta_{i} e_{1}^{(i)}$ and $\sum_{i} \sum_{j>2} a_{i j} e_{j}^{(i)}$. Similarly, if $l \in L$ fixes $u^{f}$ then it must fix both $\sum_{i} \gamma_{i} e_{3}^{(i)}$ and $\sum_{i} \sum_{j \leq 2} a_{i j} e_{j}^{(i)}$. As a consequence every element of $C_{L}\left(u^{e}, u^{f}\right)$ must also fix $\sum_{i} \sum_{j>2} a_{i j} e_{j}^{(i)}+\sum_{i} \sum_{j \leq 2} a_{i j} e_{j}^{(i)}=u$. Applying this construction to $u_{1}, \ldots, u_{b}$ we get that

$$
\left\{w_{1}, w_{2}, w_{3}, u_{1}^{e}, u_{1}^{f}, u_{2}^{e}, u_{2}^{f}, \ldots, u_{b}^{e}, u_{b}^{f}\right\}
$$

is a base of size $2 b+3$ for $H$ acting on $U_{1,0} \oplus \ldots \oplus U_{t, 0}$.

If $p \nmid k$, then there is nothing more to do, since in this case $V \simeq U_{1,0} \oplus \ldots \oplus U_{t, 0}$ as $\mathbb{F}_{q} H$-modules.

For the remainder, let $p \mid k$ and $W=W_{1} \oplus \ldots \oplus W_{t}$ where $W_{i}$ is the 1-dimensional submodule of $U_{i, 0}$ for all $i$ with $1 \leq i \leq t$. For any $x \in U$, let $\bar{x}=x+W \in U / W$ be the associated element in the factor space. Now, we claim that

$$
\left\{\bar{w}_{1}, \bar{w}_{2}, \bar{w}_{3}, \bar{u}_{1}^{e}, \bar{u}_{1}^{f}, \bar{u}_{2}^{e}, \bar{u}_{2}^{f}, \ldots, \bar{u}_{b}^{e}, \bar{u}_{b}^{f}\right\}
$$

is a base for $H$ acting on $\left(\oplus_{i} U_{i, 0}\right) / W \simeq V$. 
Let $z_{i}=\sum_{j} e_{j}^{(i)}$ for every $1 \leq i \leq t$, so $\left\{z_{1}, \ldots, z_{t}\right\}$ is a basis for $W$. An element $g \in H$ fixes $\bar{w}_{s}$ (where $s \in\{1,2,3\}$ ) if and only if there are field elements $\lambda_{1}, \ldots, \lambda_{t}$ such that $g\left(w_{s}\right)=w_{s}+\sum_{i} \lambda_{i} z_{i}$. But $g$ permutes the basis vectors in $\left\{e_{j}^{(i)} \mid 1 \leq i \leq t, 1 \leq j \leq k\right\}$ and also the subspaces $\left\{U_{i, 0} \mid 1 \leq i \leq t\right\}$. A consequence of this is that the projection of $g\left(w_{s}\right)$ to any $U_{i, 0}$ must be a non-zero linear combination of exactly two basis vectors from $\left\{e_{j}^{(i)} \mid 1 \leq j \leq k\right\}$. Since $k \geq 7$, this can happen only if $\lambda_{i}=0$ for every $1 \leq i \leq t$, i.e. when $g$ fixes $w_{s}$. So $C_{H}\left(\bar{w}_{s}\right)=C_{H}\left(w_{s}\right)$ follows for every $s$ with $1 \leq s \leq 3$. The same argument can be applied to prove that $C_{H}\left(\bar{u}_{s}^{f}\right)=C_{H}\left(u_{s}^{f}\right)$ for every $1 \leq s \leq b$.

Finally, let us assume that $g \in C_{H}\left(\bar{w}_{1}, \bar{w}_{2}, \bar{w}_{3}\right)=L$ and $g\left(\bar{u}_{s}^{e}\right)=\bar{u}_{s}^{e}$ for some $1 \leq s \leq b$. Again this means that $g\left(u_{s}^{e}\right)=u_{s}^{e}+\sum_{i} \lambda_{i} z_{i}$ for some field elements $\lambda_{1}, \ldots, \lambda_{t}$. But the linear combination we used to define $u_{s}^{e}$ contains no $e_{2}^{(i)}$ with non-zero coefficient. In other words $u_{s}^{e}$ is contained in the $L$-invariant subspace generated by $\left\{e_{j}^{(i)} \mid j \neq 2,1 \leq i \leq t\right\}$, so this must also hold for $g\left(u_{s}^{e}\right)=u_{s}^{e}+\sum_{i} \lambda_{i} z_{i}$, which implies that $\lambda_{i}=0$ for every $i$, i.e. $C_{L}\left(\bar{u}_{s}^{e}\right)=C_{L}\left(u_{s}^{e}\right)$ holds. We proved that

$$
C_{H}\left(\bar{w}_{1}, \bar{w}_{2}, \bar{w}_{3}, \bar{u}_{1}^{e}, \bar{u}_{1}^{f}, \ldots, \bar{u}_{b}^{e}, \bar{u}_{b}^{f}\right)=C_{H}\left(w_{1}, w_{2}, w_{3}, u_{1}^{e}, u_{1}^{f}, \ldots, u_{b}^{e}, u_{b}^{f}\right)=1,
$$

as claimed.

We can conclude Theorem 1.1 for alternating-induced groups.

Theorem 3.9. If $H \leq G L(V)$ is an alternating-induced linear group, then

$$
b_{V}(H) \leq 17+2 \frac{\log |H|}{\log |V|} .
$$

Proof. Again, we can assume that $k \geq 7$. By using the same notation as above let $H$ act on $U$ by permuting the basis $B=\left\{e_{j}^{(i)} \mid 1 \leq i \leq t, 1 \leq j \leq k\right\}$. This action is clearly transitive, so we can use Theorem 1.2 to conclude that we can color the basis vectors by using at most $48 \sqrt[k t]{|H|}$ colors such that only the identity of $H$ fixes this coloring, i.e. $d_{B}(H) \leq 48 \sqrt[k t]{|H|}$. Now any vector $u \in U$ can be seen as a coloring of this basis by using at most $\left|\mathbb{F}_{q}\right|=q$ colors. By (2) of Remark 2.1, it follows that

$$
b_{U}(H) \leq\left\lceil\log _{q}\left(d_{B}(H)\right)\right\rceil \leq\left\lceil\log _{q}(48 \sqrt[k t]{|H|})\right\rceil<7+\frac{\log |H|}{k t \log q}=7+\frac{\log |H|}{\log |U|} .
$$

By Lemma 3.8, $b_{V}(H) \leq 2 b_{U}(H)+3 \leq 17+2(\log |H| / \log |V|)$, as claimed.

3.3. Classical-induced representations without multiplicities. In this subsection let $q$ be a power of the prime $p, V=\oplus_{i=1}^{t} V_{i}$ be a direct sum of $\mathbb{F}_{q}$ vector spaces, and $T_{V}$ as above. We also use the notation $H_{i}, \Pi, N$ defined in Section 3.1.

Let $X: H \rightarrow G L(V(p))$ be a $\left(\bmod T_{V}\right)$-representation of $H$ such that $X(H) T_{V}$ acts on $\Pi=\left\{V_{1}, \ldots, V_{t}\right\}$ in a transitive way. By our discussion at the end of Section 3.1, this means that $X=\operatorname{Ind}_{H_{i}}^{H}\left(X_{i}\right)$, where $X_{i}: H_{i} \rightarrow \Gamma L\left(V_{i}\right)$ is a projective representation of $H_{i}$ for every $1 \leq i \leq t$. Then there is an associated homomorphism $\mathfrak{X}: H \rightarrow N_{G L(V(p))}\left(T_{V}\right) / T_{V}$ defined 
by $\mathfrak{X}(h):=X(h) T_{V} / T_{V}$. For the remainder of this subsection let $L=\mathfrak{X}(H)$ be the image of this homomorphism. Note that the action of $H$ on $\Pi$ inherits an action of $L$ on $\Pi$.

In this subsection we additionally assume that $X$ is classical-induced, i.e. the image $K_{i}$ of the homomorphism $\mathfrak{X}_{i}: H_{i} \rightarrow P \Gamma L\left(V_{i}\right)$ is some classical group i.e. $S_{i}=\operatorname{soc}\left(K_{i}\right) \leq P \Gamma L\left(V_{i}\right)$ is isomorphic to some simple classical group $S=\mathrm{Cl}\left(k, q_{0}\right) \leq P \Gamma L(k, q)$ for $k \geq 9$ where $\mathbb{F}_{q_{0}}$ is some subfield of $\mathbb{F}_{q}$. When $k \geq 9$ the group generated by all inner, diagonal and field automorphisms of $S$ (for the remainder, we denote this group by $\operatorname{IDF}(S)$ ) has index at most 2 in $\operatorname{Aut}(S)$.

We introduce some further notation. For any subset $\Delta \subseteq \Pi$ let $V_{\Delta}:=\oplus_{V_{i} \in \Delta} V_{i}$, and $X_{\Delta}$ : $N_{H}(\Delta) \rightarrow G L\left(V_{\Delta}(p)\right)$ be the $\left(\bmod T_{V_{\Delta}}\right)$-representation of $N_{H}(\Delta)$ defined by taking the restriction of $X(h)$ to $V_{\Delta}$ for all $h \in N_{H}(\Delta)$. Furthermore, let the associated homomorphism $\mathfrak{X}_{\Delta}$ be $\mathfrak{X}_{\Delta}(h):=X_{\Delta}(h) T_{V_{\Delta}} / T_{V_{\Delta}}$. Define $L_{\Delta}=\mathfrak{X}_{\Delta}\left(N_{H}(\Delta)\right)$ and $S_{\Delta}:=\operatorname{soc}\left(\mathfrak{X}_{\Delta}\left(C_{H}(\Delta)\right)\right)$. Note that $S_{\Delta}$ is a normal subgroup of $L_{\Delta}$, and if $S_{\Delta} \neq 1$, then it is a subdirect product of $|\Delta|$ copies of $S$.

We next introduce a condition which we will additionally assume in this subsection.

Multiplicity-free condition. If $\Delta \subseteq \Pi$ is an $H$-block such that $S_{\Delta} \simeq S$ and all $\mathfrak{X}_{i}: S_{\Delta} \rightarrow P \Gamma L\left(V_{i}\right)$ for $i \in \Delta$ are projectively equivalent, then $|\Delta|=1$.

A consequence of this assumption is the following.

Proposition 3.10. Let $X$ be classical-induced. Let $\Delta \subseteq \Pi$ be any $H$-block satisfying $S_{\Delta} \simeq S$. Suppose that the multiplicity-free condition holds. Then $|\Delta| \leq 2$.

Proof. First note that if $\Delta^{\prime} \subset \Delta$ is any $H$-block, then the assumption $S_{\Delta} \simeq S$ implies that $S_{\Delta^{\prime}} \simeq S$. For simpler notation, we can assume that $\Delta=\left\{V_{1}, \ldots, V_{d}\right\}$ for $d=|\Delta|$. By assumption, $S_{\Delta}$ is a diagonal subgroup of $S_{1} \times \ldots \times S_{d} \simeq S^{d}$. So, $S_{\Delta}$ can be identified with $\left\{\left(s, s^{z_{2}}, \ldots, s^{z_{d}}\right) \mid s \in S\right\}$, where $z_{2}, \ldots, z_{d} \in \operatorname{Aut}(S)$ are fixed elements. Now, if $z_{i}^{-1} z_{j} \in \operatorname{IDF}(S)$, then $\mathfrak{X}_{i}: S_{\Delta} \rightarrow P \Gamma L\left(V_{i}\right)$ and $\mathfrak{X}_{j}: S_{\Delta} \rightarrow P \Gamma L\left(V_{j}\right)$ are projectively equivalent. The relation $V_{i} \sim V_{j} \Longleftrightarrow z_{i}^{-1} z_{j} \in \operatorname{IDF}(S)$ defines an $N_{H}(\Delta)$-congruence on $\Delta$. Using that $|\operatorname{Aut}(S): \operatorname{IDF}(S)| \leq 2$ and the first sentence of the proof, we get that there is an $H$-block $\Delta^{\prime} \subset \Delta$ such that $\left|\Delta^{\prime}\right| \geq|\Delta| / 2, S_{\Delta^{\prime}} \simeq S$ and all $\mathfrak{X}_{i}: S_{\Delta^{\prime}} \rightarrow P \Gamma L\left(V_{i}\right)$ for $i \in \Delta^{\prime}$ are projectively equivalent. Thus, the result follows from the multiplicity-free condition.

For the rest of this subsection let $\Delta \subseteq \Pi$ be an $H$-block. The group $S_{\Delta}$ is either trivial or is a subdirect product of isomorphic simple classical groups. As for subdirect products of alternating groups in Section 2, this means that $S_{\Delta}$ is a direct product of diagonal subgroups corresponding to a partition $\Delta=\cup_{i} \Delta_{i}$ of $\Delta$ to equal parts. Again, we call the size of the parts of this partition the linking factor of $S_{\Delta}$. Note that the $\Delta_{i}$ themselves are $H$-blocks and $S_{\Delta_{i}} \simeq S$ holds (for each $i$ ). Hence, by Proposition 3.10, the linking factor of $S_{\Delta}$ is at most 2. As before, let $N=C_{H}(\Pi)$ be the kernel of the action of $H$ on $\Pi$.

The following follows from Theorem 3.1. 
Theorem 3.11. With the above assumptions, there exists a universal constant $c>0$ such that $b_{X_{1}}\left(K_{1}\right) \leq 18\left(\log \left|K_{1}\right|\right) /\left(\log \left|V_{1}\right|\right)+c$.

Assume that $\mathfrak{X}(N) \neq 1$. Then $\operatorname{soc}(\mathfrak{X}(N))=S_{\Pi}$ for the $H$-block $\Pi$, so $\operatorname{soc}(\mathfrak{X}(N))$ is a subdirect product of the simple classical groups $S_{i}$ with linking factor at most 2 . Thus $|N| \geq\left|S_{1}\right|^{t / 2} \geq\left|K_{1}\right|^{2 t / 5}$ (see [22, Page 18]). From this and by Theorem 3.11 we have $b_{X_{1}}\left(H_{1}\right)=b_{X_{1}}\left(K_{1}\right) \leq 45(\log |N|) /(\log |V|)+c$. A slightly modified version of Theorem 3.4 gives $b_{X}(H) \leq 45(\log |H|) /(\log |V|)+c$ for another universal constant $c>0$.

From now on assume that $\mathfrak{X}(N)=1$. This means that $L$ acts faithfully on $\Pi$. Let $M$ be a normal subgroup of $H$ strictly above $\operatorname{ker}(\mathfrak{X})$ such that $\mathfrak{X}(M)$ is a minimal normal subgroup of $L$ and let $\Delta$ be an orbit of $M$ on $\Pi$. Furthermore, let $M_{\Delta}:=\mathfrak{X}_{\Delta}(M) \triangleleft L_{\Delta}$. Notice that $\Delta \subseteq \Pi$ is an $H$-block of size at least 2 and $M_{\Delta}$ is a direct product of isomorphic simple groups.

Assume first that $S_{\Delta} \neq 1$. Then $S_{\Delta}$ is a subdirect product of the isomorphic (nonabelian) simple classical groups from the set $\left\{S_{i} \mid i \in \Delta\right\}$.

If $M_{\Delta}$ centralizes $S_{\Delta}$, then all $\mathfrak{X}_{i}: S_{\Delta} \rightarrow P \Gamma L\left(V_{i}\right)$ for $i \in \Delta$ are projectively equivalent since $M$ is transitive on $\Delta$. This contradicts our multiplicity-free assumption. So we assume that $M_{\Delta}$ does not centralize $S_{\Delta}$. Since both $M_{\Delta}$ and $S_{\Delta}$ are normal subgroups in $L_{\Delta}$, this implies that $M_{\Delta} \cap S_{\Delta} \neq 1$. In particular $M_{\Delta}$ and $M_{\Delta} \cap S_{\Delta}$ are isomorphic to some powers of the (non-abelian) simple classical group $S$. Since $M_{\Delta}$ is transitive on $\Delta$, we have that $|\Delta| \geq 5$ and $S_{\Delta}$ cannot contain a nontrivial, proper $M_{\Delta}$-invariant normal subgroup. But $M_{\Delta} \cap S_{\Delta} \neq 1$ is normal in both $M_{\Delta}$ and $S_{\Delta}$. Since any subnormal subgroup of $M_{\Delta}$ is normal in $M_{\Delta}$, we get that $S_{\Delta}$ is simple, so $S_{\Delta} \simeq S$ has linking factor $|\Delta| \geq 5$, a contradiction.

We remain with the case when $S_{\Delta}=1$. Then $L_{\Delta}$ and $M_{\Delta}$ act faithfully and transitively on $\Delta$ and $M_{\Delta}$ is a normal subgroup of $L_{\Delta}$ isomorphic to a direct product of isomorphic simple groups. By Theorem 2.8, $d_{\Delta}\left(L_{\Delta}\right) \leq 12$, or $\operatorname{Alt}(\Delta) \leq L_{\Delta} \leq \operatorname{Sym}(\Delta)$. In the former case $b_{P(\Delta)}\left(L_{\Delta}\right) \leq 4$, by $(2)$ of Remark 2.1 , and so $b_{V_{\Delta}}\left(L_{\Delta}\right) \leq 4$ (any subset of $\Delta$ can be represented by a vector in $V_{\Delta}$ whose projection to $V_{i} \in \Delta$ is non-zero if and only if $V_{i}$ is an element of the subset). Thus, $b_{V_{\Delta}}\left(N_{H}(\Delta)\right) \leq 5$. Since $H_{i} \leq N_{H}(\Delta)$ for any $V_{i} \in \Delta$, we then get the desired bound for $b_{X}(H)$ using Theorem 3.4. Assume that the latter case holds, namely that $m:=|\Delta| \geq 13$ and $\operatorname{Alt}(\Delta) \leq L_{\Delta} \leq \operatorname{Sym}(\Delta)$. In this case for any $V_{i} \in \Delta$, we have that $\mathfrak{X}_{\Delta}\left(H_{i}\right) \cong \operatorname{Alt}([m-1])$ or $\mathfrak{X}_{\Delta}\left(H_{i}\right) \cong \operatorname{Sym}([m-1])$ must hold. But $S_{i}$ is a composition factor of $\mathfrak{X}_{\Delta}\left(H_{i}\right)$ and it is a simple classical group. A contradiction.

Let us summarize the results of this subsection.

Theorem 3.12. There exists a universal constant $c>0$ such that if $X: H \rightarrow G L(V)$ is a $\left(\bmod T_{V}\right)$-representation of $H$ (with respect to some direct sum decomposition $\left.V=\oplus_{i=1}^{t} V_{i}\right)$, which is a classical-induced representation possessing the multiplicity-free condition, then $b_{X}(H) \leq 45(\log |H|) /(\log |V|)+c$.

3.4. Eliminating small tensor product factors from the $K_{i}$. Let us continue to use the notation of this section. 
The purpose of this subsection is to reduce the affine case of Theorem 1.1 to the case when each $K_{i}$ acts on $V_{i}$ either as a "big" classical group (possibly over a field extension $\mathbb{F}_{q}$ of $\mathbb{F}_{p}$ ) or as an alternating or symmetric group on the non-trivial irreducible component of its natural permutation module. More precisely, we will reduce the affine case of Theorem 1.1 to the case when the action of $H$ is alternating-induced or multiplicity-free classicalinduced. Since these types were dealt with in the previous two subsections, this reduction will complete the proof of Theorem 1.1 in the affine case.

Lemma 3.13. Let $\Lambda$ be a finite $L$-space for a finite group $L$. Consider the $L$-space $\Lambda^{l}$ (the $l$-th cartesian power of $\Lambda$ ) with the natural (coordinate-wise) action of $L$ on $\Lambda^{l}$. Then $b_{\Lambda^{l}}(L)=\left\lceil b_{\Lambda}(L) / l\right\rceil$.

Proof. Let $b^{\prime}:=b_{\Lambda}(L)$ and $\left\{x_{1}, x_{2}, \ldots, x_{b^{\prime}}\right\} \subset \Lambda$ be a minimal base for $L$ with respect to its action on $\Lambda$. Set $b:=\left\lceil b^{\prime} / l\right\rceil$. Let us define the vectors

$$
y_{1}=\left(x_{1}, x_{2}, \ldots, x_{l}\right), y_{2}=\left(x_{l+1}, x_{l+2}, \ldots, x_{2 l}\right), \ldots, y_{b}=\left(x_{(b-1) l+1}, \ldots, x_{b^{\prime}}, 0, \ldots, 0\right) \in \Lambda .
$$

It is easy to see that $\left\{y_{1}, \ldots, y_{b}\right\} \subset \Lambda^{l}$ is a minimal base for $L$ on $\Lambda^{l}$.

Now, we consider the case when the projective representation $X_{1}: H_{1} \rightarrow \Gamma L\left(V_{1}\right)$ preserves a proper tensor product decomposition $V_{1}=U_{1} \otimes W_{1}$ over $\mathbb{F}_{q}$ where $U_{1}$ and $W_{1}$ are $\mathbb{F}_{q}$ vector spaces and $2 \leq l:=\operatorname{dim}_{\mathbb{F}_{q}}\left(U_{1}\right) \leq \operatorname{dim}_{\mathbb{F}_{q}}\left(W_{1}\right)$. Using that $H$ transitively permutes the subspaces $V_{1}, \ldots, V_{t}$, it follows that each $X_{i}: H_{i} \rightarrow \Gamma L\left(V_{i}\right)$ preserves a corresponding tensor product decomposition $V_{i}=U_{i} \otimes W_{i}$.

By taking the composition of $X_{i}$ with the projection map to $W_{i}$, one can define new projective representations $Y_{i}: H_{i} \rightarrow \Gamma L\left(W_{i}\right)$. Let $Y: H \rightarrow G L(W(p))$ be the induced representation $Y=\operatorname{Ind}_{H_{1}}^{H}\left(Y_{1}\right)$, where $W$ can be identified with $W_{1} \oplus \ldots \oplus W_{t}$. The key of our reduction argument is the following lemma, which gives an upper bound for $b_{X}(H)$ in terms of $b_{Y}(H)$.

Lemma 3.14. With the above notation we have $b_{X}(H) \leq\left\lceil b_{Y}(H) / l\right\rceil+4$.

Proof. By using a construction of Liebeck and Shalev (see the proof of [30, Lemma 3.3]), for each $1 \leq i \leq t$ there exist three vectors $v_{1}^{(i)}, v_{2}^{(i)}, v_{3}^{(i)} \in V_{i}$ such that

$$
C_{G L\left(U_{i}\right) \otimes G L\left(W_{i}\right)}\left(v_{1}^{(i)}, v_{2}^{(i)}, v_{3}^{(i)}\right) \leq \operatorname{id}_{U_{i}} \otimes G L\left(W_{i}\right) .
$$

Additionally, let $v_{4}^{(i)}=\alpha v_{1}^{(i)}$ for each $1 \leq i \leq t$, where $\alpha$ is some generator of $\mathbb{F}_{q}^{\times}$. Define $v_{j}=\sum_{i=1}^{t} v_{j}^{(i)}$ for $j=1,2,3$ and let $L:=C_{H}\left(v_{1}, v_{2}, v_{3}\right)$. The choice of $v_{1}^{(i)}$ and $v_{4}^{(i)}$ guaranties that $X_{i}(L) \subset G L\left(U_{i}\right) \otimes G L\left(W_{i}\right)$ for each $i$, so $X_{i}(L) \subset \operatorname{id}_{U_{i}} \otimes G L\left(W_{i}\right)$ by the displayed formula above. It follows that the restriction map $X_{i}:\left(L \cap H_{i}\right) \rightarrow \Gamma L\left(V_{i}\right)$ is projectively equivalent to an $l=\operatorname{dim}_{\mathbb{F}_{q}} U_{i}$ multiple of $Y_{i}:\left(L \cap H_{i}\right) \rightarrow \Gamma L\left(W_{i}\right)$.

Let $\Delta_{1}, \ldots, \Delta_{s} \subset \Pi$ be the orbits of $L$ on $\Pi, V_{\Delta_{j}}=\oplus_{V_{i} \in \Delta_{j}} V_{i}$ and $W_{\Delta_{j}}=\oplus_{V_{i} \in \Delta_{j}} W_{i}$ for every $1 \leq j \leq s$. Then each $V_{\Delta_{j}}$ is $X(L)$-invariant which means that $X=\oplus_{j=1}^{s} X_{\Delta_{j}}$ on $L$, where the $\left(\bmod T_{V_{\Delta_{j}}}\right)$-representation $X_{\Delta_{j}}: L \rightarrow G L\left(V_{\Delta_{j}}(p)\right)$ is defined by taking 
the restriction of $X(L)$ to $V_{\Delta_{j}}$. One can similarly define the $\left(\bmod T_{W_{\Delta_{j}}}\right)$-representations $Y_{\Delta_{j}}: L \rightarrow G L\left(W_{\Delta_{j}}(p)\right)$ and establish the decomposition $Y=\oplus_{j=1}^{s} Y_{\Delta_{j}}$ on $L$. This means that if $V_{a} \in \Delta_{j}$ is arbitrary, then $X_{\Delta_{j}}=\operatorname{Ind}_{L \cap H_{a}}^{L}\left(X_{a}\right)$ and $Y_{\Delta_{j}}=\operatorname{Ind}_{L \cap H_{a}}^{L}\left(Y_{a}\right)$. Since $X_{a}$ on $L$ is projectively equivalent to the $l$ multiple of $Y_{a}$ on $L$, and induction of representations preserves multiplicity, we get that $X_{\Delta_{j}}$ is $\left(\bmod T_{V_{\Delta_{j}}}\right)$-equivalent to the $l$ multiple of $Y_{\Delta_{j}}$ on $L$ for every $1 \leq j \leq s$. So, $X=\oplus_{j=1}^{s} X_{\Delta_{j}}$ is $\left(\bmod T_{V}\right)$-equivalent to the $l$ multiple of $Y$ on $L$. By using Lemma 3.13, we get that $b_{X}(L)=\left\lceil b_{Y}(L) / l\right\rceil$. Since $b_{X}(H) \leq b_{X}(L)+4$ and $b_{Y}(L) \leq b_{Y}(H)$ hold trivially, the result follows.

Corollary 3.15. With the above notation, if $b_{Y}(H) \leq c_{1} \cdot \frac{\log |H|}{\log |W|}+c_{2}$ for some constants $c_{1}$ and $c_{2} \geq 10$, then $b_{X}(H) \leq c_{1} \cdot \frac{\log |H|}{\log |V|}+c_{2}$.

Proof. By Lemma 3.14 and by assumption,

$$
b_{X}(H) \leq\left\lceil\frac{b_{Y}(H)}{l}\right\rceil+4 \leq c_{1} \frac{\log |H|}{l \log |W|}+\frac{c_{2}}{l}+5 \leq c_{1} \frac{\log |H|}{\log |V|}+c_{2} .
$$

From now on we will assume that $K_{1} \leq G L\left(V_{1}\right) \simeq G L(k, p)$ is a primitive irreducible linear group with unbounded base size. We may make this assumption by Theorem 3.4.

Primitive groups of unbounded base size were characterized in [30, Theorem 2] and in [33, Theorem 1, Proposition 2]. In the following we collect some of their properties in a form which will be most convenient for us. Note that in the previously mentioned papers the authors form a theorem containing a tensor product of several linear groups, but for our purpose it is better to "pack" together all but the one with the largest dimension.

First we fix some further notation, mostly borrowed from [21]. Let $U=U_{k}(p)$ be a vector space of dimension $k$ over $\mathbb{F}_{p}$. Let $H \leq G L\left(U_{k}(p)\right)$ be a primitive linear group. Let $q=p^{f}$ be the largest power of $p$ such that one can extend scalar multiplication on $U$ to be an $\mathbb{F}_{q}$-vector space $U=U_{k / f}(q)$ such that $H \leq \Gamma L\left(U_{k / f}(q)\right) \leq G L\left(U_{k}(p)\right)$.

If $\mathbb{F}_{q_{0}}$ is a subfield of $\mathbb{F}_{q}$, then $\mathrm{Cl}\left(r, q_{0}\right) \leq G L(r, q)$ denotes a classical linear group over $\mathbb{F}_{q_{0}}$. Let $\mathrm{Cl}\left(r, q_{0}\right)^{(\infty)}$ be the last term of the derived series of $\mathrm{Cl}\left(r, q_{0}\right)$. Thus the factor group $\mathrm{Cl}\left(r, q_{0}\right)^{(\infty)} / Z\left(\mathrm{Cl}\left(r, q_{0}\right)^{(\infty)}\right)$ is a simple classical group.

Theorem 3.16 (Liebeck, Shalev [30], [33]). Let $H \leq G L\left(U_{k}(p)\right)$ be a primitive linear group of unbounded base size and $q=p^{f}$ be maximal such that $H \leq \Gamma L\left(U_{k / f}(q)\right)$. Then there is a tensor product decomposition $U=U_{1} \otimes U_{2}$ over $\mathbb{F}_{q}$ such that $1 \leq \operatorname{dim}\left(U_{1}\right)<\operatorname{dim}\left(U_{2}\right)$ and $H$ preserves this tensor product decomposition, that is, $H \leq N_{\Gamma L\left(U_{k / f}(q)\right)}\left(G L\left(U_{1}\right) \otimes G L\left(U_{2}\right)\right)$. Let $H^{0}=G L\left(U_{k / f}(q)\right) \cap H$ and let $H_{2}^{0}$ be the image of the projection of $H^{0}$ to $G L\left(U_{2}\right)$, that is, $H_{2}^{0}:=\left\{b \in G L\left(U_{2}\right) \mid \exists a \in G L\left(U_{1}\right): a \otimes b \in H^{0}\right\}$. Then one of the following holds.

(1) $H_{2}^{0} \simeq \operatorname{Sym}(m) \times \mathbb{F}_{q}^{*}$ or $\operatorname{Alt}(m) \times \mathbb{F}_{q}^{*}$ for some $m$ such that $U_{2}$ is the unique non-trivial irreducible component of the natural m-dimensional permutation representation of 
$\operatorname{Sym}(m)$. In that case $\operatorname{dim}_{\mathbb{F}_{q}}\left(U_{2}\right)=m-1$ unless $p \mid m$, when $\operatorname{dim}_{\mathbb{F}_{q}}\left(U_{2}\right)=m-2$. (We say more on this at the beginning of Section 3.2.)

(2) $H_{2}^{0}$ is a classical group $\mathrm{Cl}\left(r, q_{0}\right) \leq G L(r, q)$ over some subfield $\mathbb{F}_{q_{0}} \leq \mathbb{F}_{q}$, where $r=\operatorname{dim}_{\mathbb{F}_{q}}\left(U_{2}\right)$.

Proof. This follows at once from parts of [33, Theorem 1] and [33, Proposition 2].

Note that there is a similar characterization of primitive linear groups of large orders due to Jaikin-Zapirain and Pyber [24, Proposition 5.7].

In the following we will apply Theorem 3.16 to $K_{i} \leq G L\left(V_{i}\right)$ where $1 \leq i \leq t$. We can extend scalar multiplication on each $V_{i}$ to become an $\mathbb{F}_{q}$-vector space for some $q=p^{f}$ to get a tensor product decomposition $V_{i}=V_{i, 1} \otimes V_{i, 2}$ satisfying the statements of Theorem 3.16. This way $V=V_{s}(q)$ becomes a vector space over $\mathbb{F}_{q}\left(\right.$ where $s f=\operatorname{dim}_{\mathbb{F}_{p}}(V)$ ) and $H$ is included in $\Gamma L(s, q)$.

Now, we are ready to finish the proof of Theorem 1.1 for affine groups.

Theorem 3.17. There exists an absolute constant $c>0$ such that if $X: H \rightarrow G L(V)$ is an irreducible linear representation over $\mathbb{F}_{p}$, then

$$
b_{X}(H) \leq 45 \frac{\log |H|}{\log |V|}+c .
$$

Proof. We proceed by induction on the dimension of $V$. Note that in our reduction argument we work with the more general concept of $\left(\bmod T_{V}\right)$-representations instead of linear ones.

By a result of Liebeck and Shalev (see Theorem 3.1), we may assume that $V$ is an imprimitive $H$-module. Let $V=\oplus_{i=1}^{t} V_{i}$ be a maximal decomposition (i.e. $t$ is as large as possible) of $V$ preserved by $H, H_{i}=N_{H}\left(V_{i}\right)$ and $X_{i}: H_{i} \rightarrow G L\left(V_{i}\right)$ as before, so $X=\operatorname{Ind}_{H_{i}}^{H}\left(X_{i}\right)$ for each $i$. Because of the maximality of $t$, we have $K_{i}=X_{i}\left(H_{i}\right) \leq G L\left(V_{i}\right)$ is a primitive linear group, so Theorem 3.16 can be applied (in view of Theorem 3.4). Thus, an $\mathbb{F}_{q}$ vector space structure can be defined on each $V_{i}$ (and, as a consequence, also on $V$ ), such that $V_{i}=U_{i} \otimes W_{i}$ over $\mathbb{F}_{q}$, where $X_{i}\left(H_{i}\right)$ preserves this decomposition. Furthermore, $l:=\operatorname{dim}_{\mathbb{F}_{q}}\left(U_{i}\right)<\operatorname{dim}_{\mathbb{F}_{q}}\left(W_{i}\right)$.

First, let us assume that the tensor product decomposition $V_{i}=U_{i} \otimes W_{i}$ is proper, i.e. $l \geq 2$. Let $Y_{i}:=H_{i} \rightarrow \Gamma L\left(W_{i}\right)$ be the projective representation and $Y: H \rightarrow G L(W(p))$ be the $\left(\bmod T_{W}\right)$-representation for $W=\oplus_{i=1}^{t} W_{i}$ defined in the paragraph before Lemma 3.14 , so $Y=\operatorname{Ind}_{H_{1}}^{H}\left(Y_{1}\right)$. By induction, $b_{Y}(H) \leq 45 \frac{\log |H|}{\log |W|}+c$ for some constant $c \geq 10$, so the result follows by Corollary 3.15. So we can assume that $l=1$, which means $X$ is either alternating-induced or classical-induced by Theorem 3.16.

If $X$ is alternating-induced, then $b_{X}(H) \leq 2(\log |H| / \log |W|)+17$ by Theorem 3.9 and we are done.

In order to use Theorem 3.12 in the case when $X$ is classical-induced, we need to further reduce it to satisfy the multiplicity-free condition. For this purpose let $\Delta \subseteq \Pi$ be a maximal 
$H$-block violating the multiplicity-free condition, i.e. $S_{\Delta} \simeq S$ and the representations $X_{i}: S_{\Delta} \rightarrow \Gamma L\left(V_{i}\right)$ for $V_{i} \in \Delta$ are all projectively equivalent. Let $X_{\Delta}: N_{H}(\Delta) \rightarrow G L\left(V_{\Delta}(p)\right)$ be the $\left(\bmod T_{V_{\Delta}}\right)$-representation defined by the restriction of $X$ (where $T_{V_{\Delta}}$ is defined by the decomposition $\left.V_{\Delta}=\oplus_{V_{i} \in \Delta} V_{i}\right)$. Then $X=\operatorname{Ind}_{N_{H}(\Delta)}^{H}\left(X_{\Delta}\right)$. Furthermore, by choosing a suitable basis, $X_{\Delta}\left(N_{H}(\Delta)\right)$ is included into the Kronecker product of a group of monomial matrices and a group of matrices isomorphic to some classical group. (This follows easily from [27, Lemma 4.4.3(ii)].) This means that we have a tensor product decomposition of $V_{\Delta}=U_{\Delta} \oplus W_{\Delta}$ preserved by $X_{\Delta}\left(N_{H}(\Delta)\right)$. Taking the composition of $X_{\Delta}$ with the projections to the factors of this tensor product decomposition, we can define the maps $Y_{\Delta}: N_{H}(\Delta) \rightarrow G L\left(U_{\Delta}\right)$ and $Z_{\Delta}: N_{H}(\Delta) \rightarrow \Gamma L\left(W_{\Delta}\right)$ such that $Y_{\Delta}\left(N_{H}(\Delta)\right)$ consists of monomial matrices, while $Z_{\Delta}\left(N_{H}(\Delta)\right)$ is some classical group (modulo the group of scalar transformations). Then we can induce these representations to $H$ to get the monomial representation (with transitive permutation part) $Y=\operatorname{Ind}_{N_{H}(\Delta)}^{H}\left(Y_{\Delta}\right)$ and classical-induced representation $Z=\operatorname{Ind}_{N_{H}(\Delta)}^{H}\left(Z_{\Delta}\right)$. Note that $Z$ even satisfies the multiplicity-free condition by the maximal choice of $\Delta$. Furthermore, let $U:=\oplus_{i} U_{\Delta_{i}}, W:=\oplus_{i} W_{\Delta_{i}}$, where $\{\Delta=$ $\left.\Delta_{1}, \ldots, \Delta_{t /|\Delta|}\right\}$ is the orbit of $\Delta$ under the action of $H$ on the power set of $\Pi$. Thus, $Y: H \rightarrow G L(U(p))$ and $Z: H \rightarrow G L(W(p))$.

If $\operatorname{dim} U_{1} \geq \operatorname{dim} W_{1}$, then $b_{Y}(H) \leq \frac{\log |H|}{\log |U|}+10$ by use of Theorem 3.4 with $b=1$, so we get $b_{X}(H) \leq \frac{\log |H|}{\log |V|}+10$ by Corollary 3.15.

Similarly, if $\operatorname{dim} U_{1} \leq \operatorname{dim} W_{1}$ then $Z: H \rightarrow G L(W(p))$ is a multiplicity-free classical induced representation, so Theorem 3.12 can be applied to conclude that $b_{Z}(H) \leq 45 \frac{\log |H|}{\log |W|}+c$ for a suitable constant $c \geq 10$. Using Corollary 3.15 again, we get that $b_{X}(H) \leq 45 \frac{\log |H|}{\log |V|}+c$ holds, which completes our argument.

\section{NON-AFFINE PRIMITIVE PERMUtATION GROUPS}

Pyber's conjecture is known to be true for all non-affine primitive permutation groups. Since the explicit constants have not always been specified, we collect here the information needed to complete the proof of Theorem 1.1. In fact, in this section we show that if $G$ is a non-affine primitive permutation group of degree $n$, then $b(G)<45(\log |G| / \log n)$.

Let $G$ be a non-affine primitive permutation group acting on a finite set $\Omega$ of size $n$. The first result deals with almost simple groups.

Theorem 4.1 (Liebeck, Shalev [29]; Burness et al [10], [11], [12], [13]; Benbenishty [7]). If G is an almost simple primitive permutation group of degree $n$, then $b(G)<15(\log |G| / \log n)$.

A formula for $b(G)$ when $G$ is a primitive group of diagonal type has been obtained by Fawcett [17]. Here we will only need an upper bound.

Theorem 4.2 (Gluck, Seress, Shalev [20]; Fawcett [17]). If $G$ is a primitive permutation group of diagonal type and of degree $n$, then $b(G)<(\log |G| / \log n)+3<4(\log |G| / \log n)$. 
We remain to establish Theorem 1.1 when $G$ is a primitive permutation group of product type or of twisted wreath product type. For these types Pyber's conjecture has been proved by Burness and Seress [14]. In what follows we use the notation and assumptions of [14].

The first observation is that by the proof of [14, Theorem 4.1] it is sufficient to prove that if $G$ is of product type, then $b(G)<(45 / 2)(\log |G| / \log n)$. We will do this in what follows.

Let $G$ be a primitive permutation group of product type. Let $\Omega=\Gamma^{k}$ for some set $\Gamma$ and integer $k \geq 2$. There exists a primitive group $H \leq \operatorname{Sym}(\Gamma)$ of almost simple type or of diagonal type such that the following holds. Let the socle of $H$ be $T$. Let $P$ be the (transitive) action of $G$ on the set of the $k$ direct factors of $\operatorname{soc}(G)=T^{k}$. We have $T^{k} \leq G \leq H \curlywedge P$.

Write $\Omega=\Gamma_{1} \times \cdots \times \Gamma_{k}$ where $\Gamma_{i}=\Gamma$ for each $i$. Lemma 3.7 of [14] states that we may assume that $G$ induces $H$ on each of the $k$ factors $\Gamma_{i}$ of $\Omega$.

Next Burness and Seress define $a$ to be the integer part of $c_{1}+c_{2}(\log |P| / k)$ where $c_{1}$ and $c_{2}$ are absolute constants. By Theorem 2.2, we may take $c_{1}$ to be 7 and $c_{2}$ to be 1 . Put $r$ to be the integer part of $\log |\Gamma|$. Lemma 3.8 of [14] states that there exists a collection of points $\left\{\alpha_{1}, \ldots, \alpha_{\lceil a / r\rceil}\right\}$ in $\Omega$ with the property that an element $g=(1, \ldots, 1) p \in G$ fixes each $\alpha_{i}$ if and only if $p=1$.

In this paragraph assume that $H \leq \operatorname{Sym}(\Gamma)$ is an almost simple group. In this case $T$ is a non-abelian finite simple group. By [22, Page 18], we have $|\operatorname{Out}(T)| \leq|T|^{\alpha}$ where $\alpha=\log _{20160} 12$. As a result, $|G| \geq|T|^{k}|P| \geq|H|^{k /(1+\alpha)}|P|$. Continuing in the proof of [14, Proposition 3.9], we see that $c_{3}$ can be taken to be 15 , by use of Theorem 4.1 , and so $b$ can be taken to be the integer part of $15(\log |H| / \log |\Gamma|)$ (or the integer part of $7(\log |H| / \log |\Gamma|)$ in case $|\Gamma| \leq 7)$. This way the upper bound $\lceil a / r\rceil+b$ for the minimal base size of $G$ (presented in [14, Proposition 3.9]) can be explicitly computed. We obtain the inequality $b(G)<(45 / 2)(\log |G| / \log n)$.

Thus we may assume that $H$ is of diagonal type. Here $\operatorname{soc}(H)=T=S^{\ell}$, where $S$ is a non-abelian simple group and $\ell \geq 2$. We have $S^{\ell} \leq H \leq S^{\ell}$. $(\operatorname{Out}(S) \times Q)$ where $Q \leq \operatorname{Sym}(\ell)$ is the permutation group induced by the conjugation action of $H$ on the $\ell$ factors of $S^{\ell}$. If $\ell \leq 6$ or $\operatorname{Alt}(\ell) \not \leq Q$, then $b(G)<(\log |G| / \log n)+11$, by using Theorem 2.2 in the argument of $[14$, Case 1]. Thus we assume that $\ell \geq 7$ and $Q=\operatorname{Alt}(\ell)$ or $Q=\operatorname{Sym}(\ell)$.

Let $N$ be the kernel of the action of $G$ on the set $\left\{\Gamma_{1}, \ldots, \Gamma_{k}\right\}$. The socle $\operatorname{soc}(G)$ of $G$ is contained in $N$. Let $R$ be the preimage in $N$ of the solvable radical of $N / \operatorname{soc}(G)$. By Theorem 4.2, there exists a base $B_{1} \subseteq \Omega$ for $\operatorname{soc}(G)$ such that $\left|B_{1}\right|<4(\log |G| / \log n)$. However we would first like a base for $R$. Using the $\alpha$ above and the assumption $\ell \geq 7$, we have a base $B_{2}$ for $R$ of size less than $4.16(\log |G| / \log n)$. Assume that $R \neq N$. Consider the preimage $A$ in $N$ of the socle of $N / R$. This is a direct product of, say $k / t$ copies of a diagonal subgroup isomorphic to $\operatorname{Alt}(\ell)$. (Previously we called the integer $t$ the linking factor of $A / R$.) By $\ell \geq 7$, by use of $\alpha$, and by Theorem 4.2, we see that there exists a 
subset $B_{3}$ of $\Omega$ such that $B_{2} \cup B_{3}$ is a base for $R$ and

$$
\left|B_{3}\right|<5+4 \frac{\log |\operatorname{Alt}(\ell)|}{t \log |T|^{\ell-1}} \leq 5+4 \frac{\log |G|}{\log n} .
$$

Thus there exists a base $B_{4}$ for $N$ of size at most $8.16(\log |G| / \log n)+6$. Let $M$ be $C_{G}\left(B_{4}\right)$. This group embeds into the transitive group $G / N$ acting on the set $\left\{\Gamma_{1}, \ldots, \Gamma_{k}\right\}$. By Theorem 1.2, we have $d(M) \leq 48 \sqrt[k]{|G|}$. By (2) of Remark 2.1, we see that

$$
b_{\Omega}(M)<7+\frac{\log |G|}{k \log |\Gamma|}
$$

From these we conclude that $b(G) \leq 9.16(\log |G| / \log n)+13<(45 / 2)(\log |G| / \log n)$.

This completes the proof of Theorem 1.1.

\section{ACKNOWLEDGEMENT}

We thank Laci Pyber for drawing our attention to some of the references in this paper.

\section{REFERENCES}

[1] Albertson, M. O.; Collins, K. L. Symmetry breaking in graphs. Electron. J. Combin. 3 (1996), no. 1, RP \#18.

[2] Babai, L. On the order of uniprimitive permutation groups. Ann. of Math. (2) 113 (1981), no. 3, $553-568$.

[3] Babai, L. On the order of doubly transitive permutation groups. Invent. Math. 65 (1981/82), no. 3, 473-484.

[4] Babai, L.; Cameron, P. J.; Pálfy, P. P. On the orders of primitive groups with restricted nonabelian composition factors. J. Algebra 79 (1982), no. 1, 161-168.

[5] Bailey, R. F.; Cameron, P. J. Base size, metric dimension and other invariants of groups and graphs. Bull. Lond. Math. Soc. 43 (2011), no. 2, 209-242.

[6] Bamberg, J.; Praeger, C. E. Finite permutation groups with a transitive minimal normal subgroup. Proc. London Math. Soc. (3) 89 (2004), no. 1, 71-103.

[7] Benbenishty, C. On actions of primitive groups. Ph.D. thesis, Hebrew University, Jerusalem, 2005.

[8] Blaha, K. D. Minimum bases for permutation groups: the greedy approximation. J. Algorithms 13 (1992), no. 2, 297-306.

[9] Bochert, A. Über die Transitivitätsgrenze der Substitutionengruppen, welche die alternirende ihres Grades nicht enthalten. Math. Ann. 33 (1889), no. 4, 572-583.

[10] Burness, T. C. On base sizes for actions of finite classical groups. J. Lond. Math. Soc. (2) 75 (2007), no. 3, 545-562.

[11] Burness, T. C.; Guralnick, R. M.; Saxl, J. On base sizes for symmetric groups. Bull. Lond. Math. Soc. 43 (2011), no. 2, 386-391.

[12] Burness, T. C.; Liebeck, M. W.; Shalev, A. Base sizes for simple groups and a conjecture of Cameron. Proc. Lond. Math. Soc. (3) 98 (2009), no. 1, 116-162.

[13] Burness, T. C.; O'Brien, E. A.; Wilson, R. A. Base sizes for sporadic simple groups. Israel J. Math. 177 (2010), 307-333.

[14] Burness, T. C.; Seress, Á. On Pyber's base size conjecture. Trans. Amer. Math. Soc. 367 (2015), no. 8, 5633-5651.

[15] Cameron, P. J.; Kantor, W. M. Random permutations: some group-theoretic aspects. Combin. Probab. Comput. 2 (1993), no. 3, 257-262.

[16] Dolfi, S. Orbits of permutation groups on the power set. Arch. Math. 75 (2000), 321-327. 
[17] Fawcett, J. B. The base size of a primitive diagonal group. J. Algebra 375 (2013), 302-321.

[18] Fawcett, J. B.; Praeger, C. E. Base sizes of imprimitive linear groups and orbits of general linear groups on spanning tuples. Arch. Math. (Basel) 106 (2016), no. 4, 305-314.

[19] Gluck, D.; Magaard, K. Base sizes and regular orbits for coprime affine permutation groups. J. London Math. Soc. (2) $\mathbf{5 8}$ (1998), 603-618.

[20] Gluck, D.; Seress, Á.; Shalev, A. Bases for primitive permutation groups and a conjecture of Babai. J. Algebra 199 (1998), no. 2, 367-378.

[21] Guidici, M.; Liebeck, M. W.; Praeger, C. E.; Saxl, J; Tiep, P. H. Arithmetic results on orbits of linear groups. Trans. Amer. Math. Soc. 368 (2016), 2415-2467.

[22] Guralnick, R. M.; Maróti, A.; Pyber, L. Normalizers of primitive permutation groups. ArXiv: 1603.00187 .

[23] Halasi, Z.; Maróti, A. The minimal base size for a p-solvable linear group. Proc. Amer. Math. Soc. 144 (2016), 3231-3242.

[24] Jaikin-Zapirain, A.; Pyber, L. Random generation of finite and profinite groups and group enumeration. Ann. of Math. (2) 173 (2011), no. 2, 769-814.

[25] James, J. P. Two point stabilisers of partition actions of linear groups. J. Algebra 297 (2006), no. 2, 453-469.

[26] James, J. P. Partition actions of symmetric groups and regular bipartite graphs. Bull. London Math. Soc. 38 (2006), no. 2, 224-232.

[27] Kleidman, P.; Liebeck, M. W. The subgroup structure of the finite classical groups, LMS Lecture Note Series, 129. Cambridge University Press, Cambridge, 1990.

[28] Liebeck, M. W. On minimal degrees and base sizes of primitive permutation groups. Arch. Math. (Basel) 43 (1984), no. 1, 11-15.

[29] Liebeck, M. W.; Shalev, A. Simple groups, permutation groups, and probability. J. Amer. Math. Soc. 12 (1999), no. 2, 497-520.

[30] Liebeck, M. W.; Shalev, A. Bases of primitive linear groups. J. Algebra 252 (2002), 95-113.

[31] Liebeck, M. W.; Shalev, A. Bases of primitive permutation groups. Groups, combinatorics \& geometry (Durham, 2001), 147-154, World Sci. Publ., River Edge, NJ, 2003.

[32] Liebeck, M. W.; Shalev, A. Character degrees and random walks in finite groups of Lie type. Proc. London Math. Soc. (3) 90 (2005), no. 1, 61-86.

[33] Liebeck, M. W.; Shalev, A. Bases of primitive linear groups II. J. Algebra 403 (2014), 223-228.

[34] Pyber, L. On the orders of doubly transitive permutation groups, elementary estimates. J. Combin. Theory Ser. A 62 (1993), no. 2, 361-366.

[35] Pyber, L. Asymptotic results for permutation groups, Groups and computation, DIMACS Ser. Discrete Math. Theoret. Comput. Sci. 11 (ed. L. Finkelstein and W. M. Kantor) Amer. Math. Soc. Providence RI 1993, pp. 197-219.

[36] Robinson, G. R. On the base size and rank of a primitive permutation group. J. Algebra 187 (1997), no. $1,320-321$.

[37] Scott, L. L. Representations in characteristic $p$. The Santa Cruz Conference on Finite Groups (Univ. California, Santa Cruz, Calif., 1979), pp. 319-331, Proc. Sympos. Pure Math., 37, Amer. Math. Soc., Providence, R.I., 1980.

[38] Seress, Á. The minimal base size of primitive solvable permutation groups. J. Lond. Math. Soc. (2) 53 (1996), no. 2, 243-255.

[39] Seress, Á. Primitive groups with no regular orbits on the set of subsets. Bull. London Math. Soc. 29 (1997), 697-704.

[40] Seress, Á. Permutation group algorithms. Cambridge Tracts in Mathematics, 152. Cambridge University Press, Cambridge, 2003.

[41] Sun, X.; Wilmes, J. Structure and automorphisms of primitive coherent configurations. ArXiv:1510.02195. 
Department of Mathematics, Central European University, Nádor utca 9., H-1051, BuDAPEST, Hungary

E-mail address: duyan_hulya@phd.ceu.edu

Department of Algebra and Number Theory, EÖtvös University, PÁzmány Péter sétány 1/C, H-1117, Budapest, Hungary

E-mail address: zhalasi@cs.elte.hu and halasi.zoltan@renyi.mta.hu

Alfréd Rényi Institute of Mathematics, Hungarian Academy of Sciences, Reáltanoda utca 13-15, H-1053, Budapest, Hungary

E-mail address: maroti.attila@renyi.mta.hu 